GEOLOGICA BALCANICA 50 (3), Sofia, December 2021, pp. 37-53.

\title{
Use of multivariate statistical methods to determine spatial distributions of chemical elements in soils (Mariovo region, North Macedonia)
}

\author{
Trajče Stafilov ${ }^{*}$, Robert Šajn², Danica Damčevska ${ }^{1}$, Claudiu Tănăselia ${ }^{3}$ \\ ${ }^{1}$ Institute of Chemistry, Faculty of Natural Sciences and Mathematics, Sts Cyril and Methodius University, 5 Arhimedova, \\ 1000 Skopje, North Macedonia; e-mail: trajcest@pmf.ukim.mk \\ ${ }^{2}$ Geological Survey of Slovenia, 14 Dimičeva, 1000 Ljubljana, Slovenia; e-mail: Robert.Sajn@geo-zs.si \\ ${ }^{3}$ INCDO-INOE 2000 Research Institute for Analytical Instrumentation (ICIA), Cluj-Napoca, Romania: \\ e-mail: claudiu@tanaselia.ro \\ * corresponding author
}

(Received: 20 September 2021; accepted in revised form: 23 November 2021)

\begin{abstract}
The main objective of this study is to present the distribution of different chemical elements in soil samples from the Mariovo region, North Macedonia. To determine the level of presence of chemical elements, soil samples were collected from a total of 33 locations in a $5 \times 5 \mathrm{~km}$ area. From each location, two samples of soil were collected: topsoil $(0-5 \mathrm{~cm})$ and bottom soil $(20-30 \mathrm{~cm})$. The analyses of soil samples were used to assess the prevalence of the chemical elements and the presence of heavy metals in the studied area. The analysis was carried out, using two instrumental methods: atomic emission spectrometry with inductively coupled plasma (ICP-AES) for macro-elements, and mass spectrometry with inductively coupled plasma (ICP-MS) for trace elements. A factor analysis was applied to reveal the relationships between the investigated variables (chemical elements). From the obtained results, spatial distribution maps of factors for the separate groups were prepared, as well as distribution maps for the analyzed elements. It was found that the distributions of most elements follow the lithology of the examined area. Factor analysis revealed the relationships between the investigated variables and could provide dimensionality reduction based on them.
\end{abstract}

Stafilov, T., Šajn, R., Damčevska, D., Tănăselia, C. 2021. Use of multivariate statistical methods to determine spatial distributions of chemical elements in soils (Mariovo region, North Macedonia). Geologica Balcanica 50 (3), 37-53.

Keywords: soil, chemical elements, spatial distribution, statistical analysis, Mariovo Region, North Macedonia.

\section{INTRODUCTION}

Potentially toxic elements (PTEs) are commonly found as contaminants in soil, water and food, and are carried in the air. In recent years, environmental pollution has often been associated with PTEs (Salminen et al., 2005; Davidson et al., 2006; Kabata-Pendias and Mukherjee, 2007; Reimann et al., 2011; Salomons et al., 2012; Wang et al., 2015; Stafilov and Šajn, 2019). The pollution with PTEs of soil, water and air is an important part that should never be overlooked. The main cause of emission are anthropogenic sources, especially mining and metallurgical activities (Peplow, 1999; Diawara et al., 2006; Stafilov et al., 2010a, b; Acton, 2013). For a very long time after the cessation of mining activities, the emitted metals survive in the environment and the metal contamination that occurs as a result of ore mining persists for hundreds of years after mining has ceased (Peplow, 1999).

On the basis of our previously obtained data from the whole territory of North Macedonia, soil pollu- 
tions were found in the areas of mining, metallurgical and urban activities due to the emission of certain heavy metals (As, Cd, Cu, Mn, Ni, Sb, Zn) (Stafilov et al., 2010a, b, 2013, 2018, 2019; Balabanova et al., 2013; Serafimovska et al., 2013; Bačeva et al., 2014; Stafilov and Šajn, 2016, 2019). A need arose to determine the distributions of various chemical elements in the area of the Mariovo region, situated in the central-southern part of the country. For that purpose, soil samples were collected from the whole area of the Skopje region within a grid of $5 \times 5 \mathrm{~km}$, from a total of 33 locations. From each location, two samples of soil were collected: samples of topsoil $(0-5 \mathrm{~cm})$ and samples from a deeper layer, bottom soil $(20-30 \mathrm{~cm})$. The analyses were carried out using two instrumental methods: atomic emission spectrometry with inductively coupled plasma (ICP-AES) for macro-elements and mass spectro- metry with inductively coupled plasma (ICP-MS) for the analysis of trace elements. A factor analysis was applied to analyze relations between a number of variables.

The obtained results for the content of the examined elements were statistically processed by multivariate factor analysis and cluster analysis and, based on them, spatial distribution maps were prepared for each of the factors.

\section{MATERIAL AND METHODS}

\section{Study area}

The Mariovo region is located in the central-southern part of North Macedonia and occupies the middle catchment area of the Crna River (Fig. 1). It is surrounded by vast mountain massifs: Nidže and

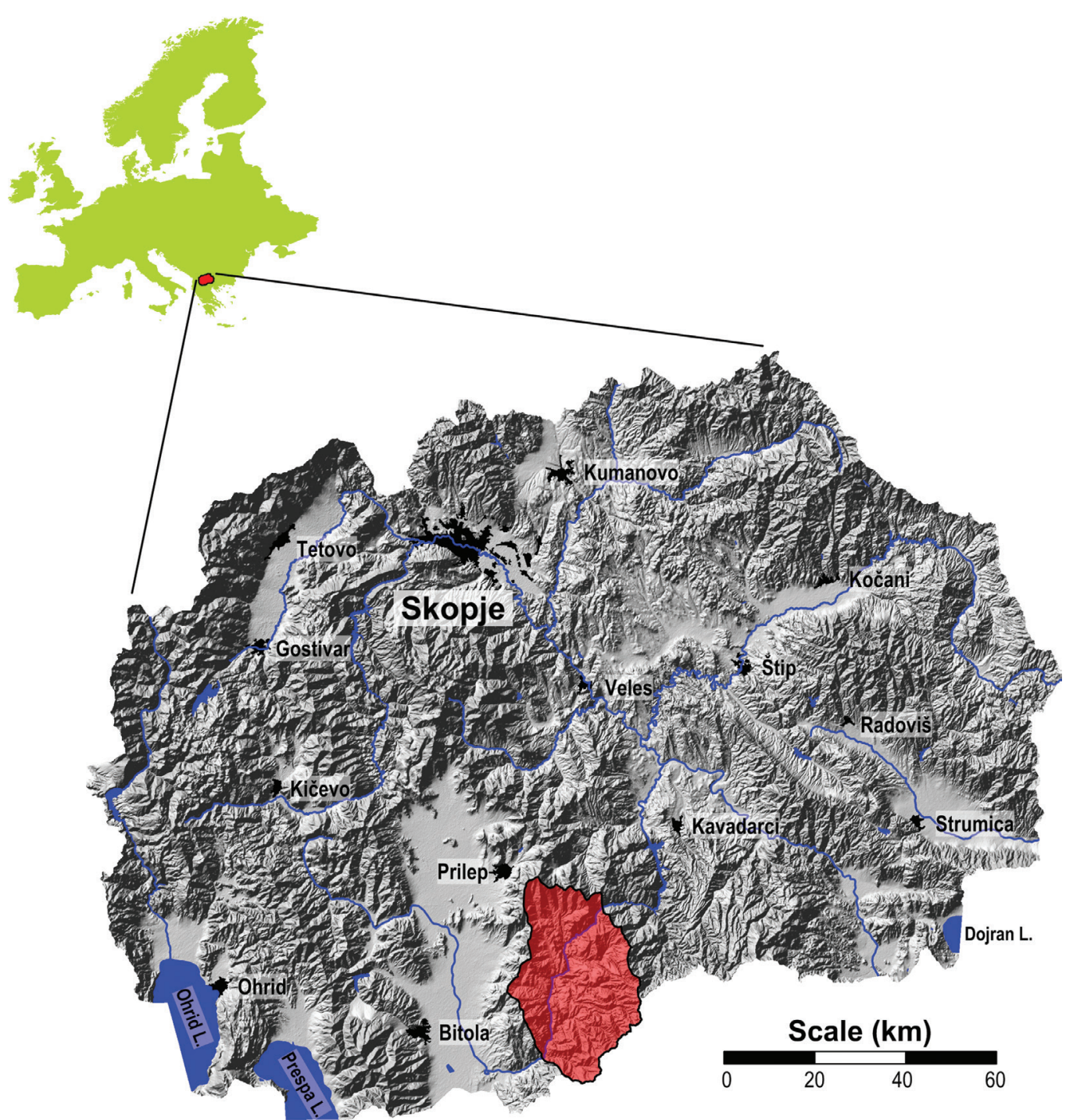

Fig. 1. Location of the studied area. 
Kožuf from the south, the Selečka Mountain from the west, the Dren Mountain from the north and Kozjak with the branches of the Kožuf Mountain from the east. The borders of Mariovo mainly trace the ridges of the mentioned mountains and the region is located between $40^{\circ} 32^{\prime} 56^{\prime \prime}$ and $41^{\circ} 15^{\prime} 07^{\prime \prime}$ north latitude and $22^{\circ} 32^{\prime} 56^{\prime \prime}$ and $22^{\circ} 04^{\prime} 15^{\prime \prime}$ east longitude (Koteski, 2013). Within these borders, the Mariovo region covers an area of $1,038 \mathrm{~km}^{2}$ and represents $4 \%$ of the total territory of the Republic of North Macedonia, with an average altitude of 1,090 m (Fig. 2). As shown in Fig. 2, the altitude around the catchments of the Crna River ranges from $400 \mathrm{~m}$ to $800 \mathrm{~m}$; in the southern part, where the Nidže Mountain is located, the altitude reaches 2200 m; and in the east, where the Kožuf Mountain massif stretches, the altitude is from $1400 \mathrm{~m}$ to $1800 \mathrm{~m}$. Most of the relief follows the Crna Riv-

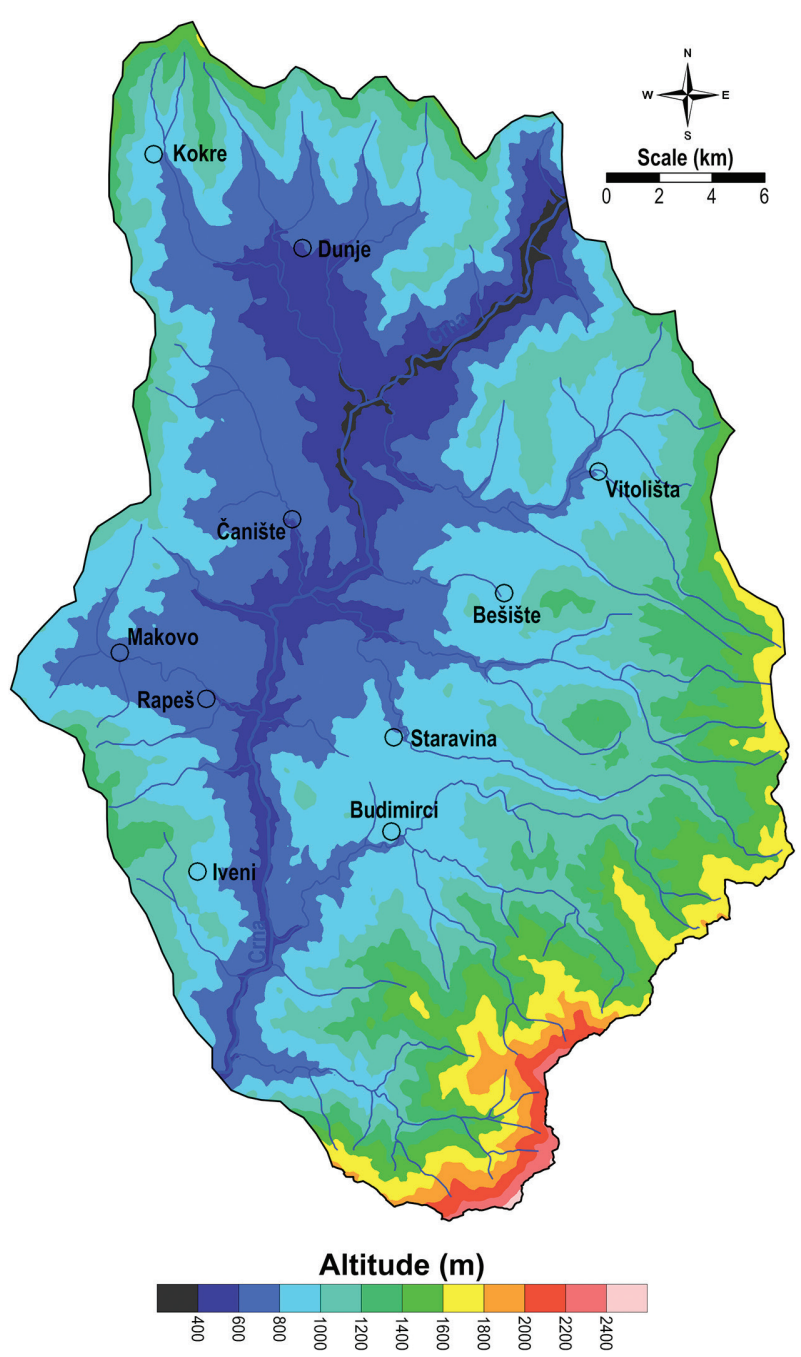

Fig. 2. The studied area according to the altitude. er, which flows in a N-S direction. The Mariovo region has a diverse relief transitioning from high mountainous to hilly to high valley. There are three tectonic morphological units: high mountains and valleys; eruptive erosive areas; and the bottom of the Mariovo Valley, which is partially flat and intersected by watercourses (Koteski, 2013; Temovski, 2013).

According to meteorological data, Mariovo is characterized by three climatic zones (Lazarevski, 1993): modified Mediterranean climate (Crna River Valley and in a narrow belt to Tikveš) with maximum rainfall in May and November, and with a minimum in summer; the hilly and terraced part of this area has a moderate continental climate, similar to the climate in the Pelagonia valleys with maximum rainfall in November, minimum in May, and the driest months are July and August; and mountain climatic zone that occupies the area from the slopes of the mountain sides of the Nidže, Kožuf and Selečka mountains.

The Mariovo region includes various geological formations of different ages. A part of this region, together with a part of the Kozjak Mountain, is in the Vardar Zone, whereas the western areas are in the Pelagonian massif (Dumurdžanov et al., 2003, 2004, 2013; Temovski, 2013; Stafilov and Šajn, 2016, Petrušev et al., 2021). The southern and central parts of the region are composed of gneisses and felsic plutonites of Proterozoic age (Fig. 3). Limited occurrence of Proterozoic shales is also present. The northern part of the Mariovo region has also Proterozoic gneisses and plutonites, while in the northeastern part Proterozoic carbonates are present. The eastern area of the region shows quite interesting geology, as it is a mixture of various geological formations. Proterozoic carbonates and gneisses are mostly present to the southeast, but Mesozoic mafic rocks and Neogene pyroclastites and volcanites also occur. In the central-eastern part, the interweaving of the following geological formations is characteristic: Neogene clastites, volcanites and pyroclastites, Paleozoic shales and Proterozoic carbonates. Proterozoic gneisses are most common along the Crna River, except for the northern part of its course, where there are Proterozoic carbonates (Fig. 3).

In terms of pedological structure, the Mariovo Valley has a heterogeneous composition that is influenced by climate, geological, biological and anthropogenic factors. The most common soil types are: ranker, regosol, cambisol, cambisol (limestonedolomite), latosol and other (Koteski et al., 2012; Filipovski et al., 2015). 




Fig. 3. Geological map of the studied area.

\section{Soil sampling and digestion}

Samples of surface soils from the Skopje region, North Macedonia, were collected according to the European guidelines and also according to our experience (Salminen et al., 2005; Stafilov et al., 2010b, Stafilov and Šajn, 2019). The study area $\left(1812 \mathrm{~km}^{2}\right)$ was covered by a sampling grid of $5 \times 5 \mathrm{~km}$ (Fig. 4). Altogether, 66 soil samples were collected from 33 locations. To distinguish possible anthropogenic pollution of the surface soil, samples from two layers were collected, topsoil $(0-5 \mathrm{~cm})$ and subsoil $(20-30 \mathrm{~cm})$. To obtain representative composite samples, five subsamples from each location on a square plot of $10 \times 10 \mathrm{~m}$ were collected. The soil samples brought to the laboratory were cleaned from plant material and stones and homogenized, then dried at room temperature. Subsequently, they were passed through a $2 \mathrm{~mm}$ sieve and ground in a porcelain mortar until reaching a final particle size below $125 \mu \mathrm{m}$. For the digestion of soil samples, open wet digestion with a mixture of acids was applied $\left(\mathrm{HNO}_{3}, \mathrm{HF}, \mathrm{HClO}_{4}\right.$ and $\left.\mathrm{HCl}\right)$ according to the ISO Standard (ISO 14869-1:2001).

\section{Instrumentation}

All samples were analysed by ICP-AES (Varian, model 715-ES) for the elements with high contents (Balabanova et al., 2010). Trace elements were analysed by ICP-MS measurements on a SCIEX Perkin Elmer Elan DRC II (Canada) inductively coupled plasma mass spectrometer with quadruple and single detector setup (Bačeva et al., 2012). Quality control of the two applied techniques was performed, using the standard addition method, and the recovery for the investigated elements ranged from $98.2 \%$ to $100.8 \%$. The quality control was also performed by the analysis of certified reference soil and geologi-

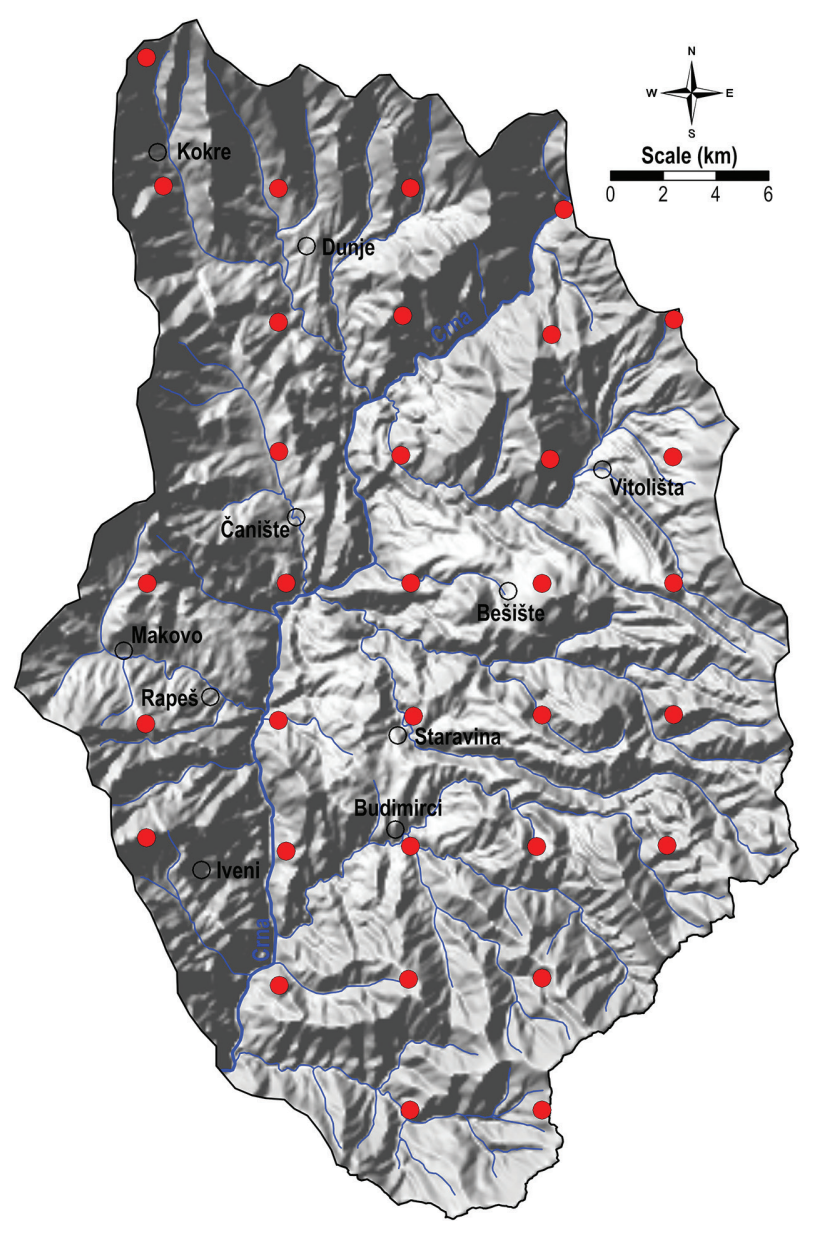

Fig. 4. Sample locations. 
cal samples: soil sample JSAC 0401 (The Japan Society for Analytical Chemistry), rock CRM samples from undersaturated igneous rock SARM 3 NIM-L Lujaurite (SA Bureau of Standards, Pretoria, S. Africa), and rock NCS DC71306 (GBW07114) (China National Analysis Centre). The values for Al, Ca, Fe, $\mathrm{K}, \mathrm{Mg}, \mathrm{Na}$ and Ti are given in per cent, values for $\mathrm{Cd}$, $\mathrm{I}$, and In in $\mu \mathrm{g} / \mathrm{kg}$, while the values for the content of the remaining elements are given in $\mathrm{mg} / \mathrm{kg}$.

\section{Data processing}

Data analysis and maps were done on a Statistica (ver. 13), QGIS (ver. 3.14) and Surfer (ver. 20) software. All field observations, analytical data and measurements were introduced into the data matrix. Parametric and non-parametric statistical methods were used for data analysis. Box-Cox transformation was used to acquire normal distributions.

Multivariate R-mode factor analysis was used to reveal associations of the chemical elements (Facchinelli et al., 2001). From numerous variables, the factor analysis (FA) derives a smaller number of new, synthetic variables. The factors contain significant information about the original variables, and they may have certain meanings. Factor analysis was performed on variables standardized to a mean of zero and one unit of standard deviation (Reimann et al., 2002). As a measure of similarity between variables, the product-moment correlation coefficient (r) was applied. For orthogonal rotation, the varimax method was used.

The universal kriging method with linear variogram interpolation was applied for the construction of the areal distribution maps of the analyzed elements and the obtained factor scores. Seven classes of the following percentile values were selected: $0-10,10-25,25-40,40-60,60-75,75-90$ and $90-100$.

\section{RESULTS AND DISCUSSION}

The soil samples were collected according to the $5 \times 5 \mathrm{~km}$ sampling network. Each sampling point describes the following 14 parameters: ID, location, soil layer, longitude (WGS84), latitude (WGS84), region, municipality, land use, geological unit, pedological unit, altitude $(\mathrm{m})$, slope $\left({ }^{\circ}\right)$, exposition $\left({ }^{\circ}\right)$, average annual temperature $\left({ }^{\circ} \mathrm{C}\right)$ and average annual precipitation (mm). Basic data of sampling points are presented in Table 1.

As a result of the analysis of the soil samples, data were obtained on the content of a total of 58 elements, from which 16 elements present in higher content were analyzed by ICP-AES ( $\mathrm{Al}, \mathrm{Ca}, \mathrm{Cr}, \mathrm{Cu}$, $\mathrm{Fe}, \mathrm{K}, \mathrm{Li}, \mathrm{Mg}, \mathrm{Mn}, \mathrm{Na}, \mathrm{Ni}, \mathrm{P}, \mathrm{Pb}, \mathrm{Sr}, \mathrm{V}$ and $\mathrm{Zn}$ ) and 42 trace elements by ICP-MS (Ag, As, Ba, Be, Bi, $\mathrm{Br}, \mathrm{Cd}$, Ce, Co, Cs, Dy, Er, Eu, Ga, Gd, Ge, Hf, Ho, I, In, La, Lu, Mo, Nb, Nd, Pd, Pr, Pt, Rb, Sb, Sc, Sm, Sn, Ta, Tb, Ti, Tl, Tm, W, Y, Yb and Zr). The descriptive statistics for the content of the elements in surface and bottom soil samples are presented in Tables 2 and 3, respectively.

Basic descriptive statistics were used for the statistical processing of the values obtained from this study. From the results obtained by applying descriptive statistics, it can be noticed that no serious deviation of the examined elements was found. The obtained values are within the average range, from which it can be determined that the presence of the investigated elements in the soil samples reflects the lithology. There is no significant difference between the obtained values and the geological composition (Stafilov et al., 2019, Stafilov and Šajn, 2019).

Most of the elements that were analyzed are within the limits of Dutch soil standards, but some elements exceed these values. The order of the distribution of contents of the major elements (Al, Ca, $\mathrm{Fe}, \mathrm{K}, \mathrm{Mg}, \mathrm{Na}$ and $\mathrm{Ti}$ ) were in the following ranges: $0.48-57.6 \% \mathrm{Al} ; 0.29-17 \% \mathrm{Ca} ; 0.18-4.8 \% \mathrm{Fe}$; $0.03-2.4 \% \mathrm{~K}$; $0.17-8.8 \% \mathrm{Mg}, 0.03-1.9 \% \mathrm{Na}$ and $0.01-0.73 \%$ Ti for topsoil $(0-5 \mathrm{~cm})$ and $0.12-2.4 \%$ $\mathrm{Al} ; 0.25-18 \% \mathrm{Ca} ; 0.029-5.0 \% \mathrm{Fe}$; $0.022-2.2 \mathrm{~K}$; $0.15-7.4 \% \mathrm{Mg}, 0.042-1.9 \% \mathrm{Na}$ and $0.024-0.42 \mathrm{Ti}$ for bottom soil $(20-30 \mathrm{~cm})$. The content of major elements is most frequently a result of the dominant geological formations of the area: Proterozoic gneisses, felsic plutonites and carbonates, and Neogene felsic pyroclastites and clastic sediments (Fig. 3).

The ratio of the contents was between the topsoil and the subsoil, and is represented in Table 4. No significant differences were found, varying the ratio of 0.66 for $\mathrm{Br}$ to 1.36 for $\mathrm{Pb}$, showing the absence of influence of possible anthropogenic activities.

A comparative analysis of the contents of the analyzed elements in topsoils from the Skopje region and the soil from the city of Skopje (urban zone), soil from North Macedonia (Stafilov and Šajn, 2016, 2019) and Europe (Salminen et al., 2005) is given in Table 5. It was found that for many elements there are differences in the content in soil from the Mariovo region compared with those from Macedonian soil as well as with the European soil (Table 5), showing their dependence on the specific lithogenic origin of the rocks in the separate sub-regions of the 
Table 1

Basic information about sample locations

\begin{tabular}{|c|c|c|c|c|c|c|c|}
\hline Location & $\begin{array}{l}\text { Longitude } \\
\text { (WGS84) }\end{array}$ & $\begin{array}{c}\text { Latitude } \\
\text { (WGS 84) }\end{array}$ & Land use & $\begin{array}{l}\text { Lithology } \\
\text { unit }\end{array}$ & $\begin{array}{c}\text { Chronology } \\
\text { unit }\end{array}$ & $\begin{array}{c}\text { Pedology } \\
\text { unit }\end{array}$ & $\begin{array}{l}\text { Altitude } \\
(\mathrm{m})\end{array}$ \\
\hline M-1 & 21.73750 & 40.96250 & Forest & Metamorphic r. & Proterozoic & Cambisol & 1167 \\
\hline M-2 & 21.79695 & 40.96222 & Scrub & Metamorphic r. & Proterozoic & Ranker & 2097 \\
\hline M-3 & 21.67889 & 41.00556 & Forest & Metamorphic r. & Proterozoic & Regosol & 948 \\
\hline M-4 & 21.73722 & 41.00723 & Forest & Metamorphic r. & Proterozoic & Cambisol & 1474 \\
\hline M-5 & 21.79750 & 41.00722 & Forest & Metamorphic r. & Proterozoic & Cambisol & 1471 \\
\hline M-6 & 21.61967 & 41.05334 & Scrub & Metamorphic r. & Proterozoic & Ranker & 1252 \\
\hline M-7 & 21.68250 & 41.05139 & Forest & Metamorphic r. & Proterozoic & Regosol & 687 \\
\hline M-8 & 21.73861 & 41.05278 & Scrub & Metamorphic r. & Proterozoic & Regosol & 822 \\
\hline M-9 & 21.79556 & 41.05222 & Forest & Carbonates & Proterozoic & Cambisol & 1118 \\
\hline M-10 & 21.85445 & 41.05222 & Forest & Magmatic r. & Neogene & Cambisol & 1349 \\
\hline M-11 & 21.61570 & 41.09836 & Scrub & Metamorphic r. & Proterozoic & Ranker & 875 \\
\hline M-12 & 21.67944 & 41.09611 & Grassland & Metamorphic r. & Proterozoic & Regosol & 539 \\
\hline M-13 & 21.74055 & 41.09722 & Grassland & Metamorphic r. & Proterozoic & Vertisol & 854 \\
\hline M-14 & 21.79861 & 41.09722 & Scrub & Carbonates & Proterozoic & Cambisol & 979 \\
\hline M-15 & 21.85805 & 41.09695 & Forest & Clastites & Mesozoic & Cambisol & 1278 \\
\hline M-16 & 21.62053 & 41.14339 & Pastures & Metamorphic r. & Proterozoic & Regosol & 846 \\
\hline M-17 & 21.68333 & 41.14306 & Pastures & Metamorphic r. & Proterozoic & Regosol & 602 \\
\hline M-18 & 21.73973 & 41.14278 & Forest & Carbonates & Neogene & Ranker & 716 \\
\hline M-19 & 21.79917 & 41.14222 & Pastures & Carbonates & Paleozoic & Litosol & 1051 \\
\hline M-20 & 21.85861 & 41.14195 & Pastures & Metamorphic r. & Proterozoic & Cambisol & 1249 \\
\hline M-21 & 21.68056 & 41.18806 & Pastures & Magmatic r. & Proterozoic & Ranker & 682 \\
\hline M-22 & 21.73583 & 41.18639 & Agricultural area & Metamorphic r. & Proterozoic & Ranker & 579 \\
\hline M-23 & 21.80333 & 41.18472 & Scrub & Carbonates & Mesozoic & Litosol & 1108 \\
\hline M-24 & 21.85889 & 41.18500 & Scrub & Metamorphic r. & Proterozoic & Cambisol & 1060 \\
\hline M-25 & 21.68083 & 41.23222 & Pastures & Metamorphic r. & Proterozoic & Regosol & 544 \\
\hline M-26 & 21.73722 & 41.23416 & Scrub & Metamorphic r. & Proterozoic & Ranker & 783 \\
\hline M-27 & 21.80444 & 41.22722 & Forest & Carbonates & Mesozoic & Cambisol & 920 \\
\hline M-28 & 21.86001 & 41.23194 & Forest & Metamorphic r. & Proterozoic & Regosol & 1279 \\
\hline M-29 & 21.62917 & 41.27917 & Grassland & Magmatic r. & Proterozoic & Ranker & 792 \\
\hline M-30 & 21.68139 & 41.27805 & Grassland & Metamorphic r. & Proterozoic & Regosol & 682 \\
\hline M-31 & 21.74111 & 41.27778 & Scrub & Metamorphic r. & Proterozoic & Litosol & 886 \\
\hline M-32 & 21.81055 & 41.27000 & Forest & Clastites & Mesozoic & Cambisol & 319 \\
\hline M-33 & 21.62194 & 41.32306 & Grassland & Magmatic r. & Proterozoic & Ranker & 1338 \\
\hline
\end{tabular}

area. It is important to note that, except in the case of $\mathrm{Ba}$, the median value for all elements included in The New Dutchlist (http://www.contaminatedland. co.uk), are below the target values, while in some parts of the region some of the elements exceeded the target values ( $\mathrm{As}, \mathrm{Co}, \mathrm{Cr}, \mathrm{Ni}$ and $\mathrm{Pb}$ ) or even the action values (As, $\mathrm{Ba}, \mathrm{Cr}$ and $\mathrm{Ni}$ ), which is due to the lithogenic origin of these elements in soil from the Vardar tectonic zone (Stafilov et al., 2010b; Stafilov and Šajn, 2016, 2019).

To determine the degree of correlation between the elements in the samples from river sediments and soils across the whole river basin, a bivariate statistic was used, where strong correlation between the investigated elements is apparent when the absolute value of the correlation coefficient is from 0.7 to 0.9 , while good correlation is found when the correlation coefficient is between 0.5 and 0.7 (Table 6).

In factor analysis, the distribution is reduced to three synthetic variables (F1-F3). As it can be seen from the results obtained by factor analysis (Table 7), three factors (i.e., associations of elements) were obtained: $\mathrm{F} 1$ (Fe, V, Cr, Co, Ni, Mn, $\mathrm{Cu}, \mathrm{Al}, \mathrm{Sc}$, Ti, Li, Zn and In); F2 (Pd, As, Zr, Pt, Ag, Hf, Br and $\mathrm{Cs}$ ); and F3 (Ga, Rb, Be, K, Na, Nb and Ge). A total of 68 elements were reduced by factor analysis to 28 elements, which had a total factor loading of $72 \%$ of the variability (Table 7). The rest of the elements were eliminated from further analysis, because they had a low share of communality or a low tendency to form independent factors. Multivariate cluster analysis was also applied to determine the significance of the factor analysis and the 
Table 2

Descriptive statistics for the content of the elements in topsoil $(0-5 \mathrm{~cm}), n=33$

\begin{tabular}{|c|c|c|c|c|c|c|c|c|c|c|c|c|c|c|}
\hline & Unit & $\mathrm{N}$ & $\mathrm{X}$ & $\mathrm{X}_{(\mathrm{BC})}$ & $\mathrm{Md}$ & Min & Max & S & $\mathrm{S}_{\mathrm{X}}$ & $\mathrm{CV}$ & A & E & $\mathrm{A}_{(\mathrm{BC})}$ & $\mathrm{E}_{(\mathrm{BC})}$ \\
\hline $\mathrm{Ag}$ & $\mathrm{mg} / \mathrm{kg}$ & 33 & 0.72 & 0.64 & 0.51 & 0.12 & 1.9 & 0.48 & 0.083 & 66 & 0.93 & 0.03 & 0.35 & -0.75 \\
\hline $\mathrm{Al}$ & $\%$ & 33 & 2.4 & 2.3 & 2.2 & 0.19 & 5.6 & 1.2 & 0.21 & 2100 & 1.00 & 1.18 & 0.23 & 1.04 \\
\hline As & $\mathrm{mg} / \mathrm{kg}$ & 33 & 20 & 13 & 13 & 3.7 & 100 & 22 & 3.8 & 110 & 2.47 & 6.52 & 0.14 & -0.58 \\
\hline $\mathrm{Ba}$ & $\mathrm{mg} / \mathrm{kg}$ & 33 & 820 & 760 & 740 & 52 & 2000 & 440 & 76 & 54 & 0.73 & 0.87 & -0.27 & 0.88 \\
\hline $\mathrm{Be}$ & $\mathrm{mg} / \mathrm{kg}$ & 33 & 2.1 & 2.0 & 2.0 & 0.005 & 6.7 & 1.2 & 0.21 & 58 & 1.57 & 5.45 & 0.46 & 3.17 \\
\hline $\mathrm{Bi}$ & $\mathrm{mg} / \mathrm{kg}$ & 31 & 0.25 & 0.18 & 0.17 & 0.070 & 1.4 & 0.25 & 0.044 & 99 & 3.68 & 15.58 & 0.04 & 0.28 \\
\hline $\mathrm{Br}$ & $\mathrm{mg} / \mathrm{kg}$ & 31 & 2.4 & 1.5 & 1.5 & 0.005 & 18 & 3.2 & 0.58 & 130 & 3.69 & 16.66 & -0.21 & 1.34 \\
\hline $\mathrm{Ca}$ & $\%$ & 33 & 3.1 & 1.3 & 1.3 & 0.29 & 17 & 4.8 & 0.84 & 8400 & 2.24 & 3.59 & 0.09 & 0.38 \\
\hline $\mathrm{Cd}$ & $\mu \mathrm{g} / \mathrm{kg}$ & 33 & 100 & 33 & 35 & 5.0 & 730 & 140 & 25 & 140 & 2.84 & 10.85 & -0.26 & -1.24 \\
\hline $\mathrm{Ce}$ & $\mathrm{mg} / \mathrm{kg}$ & 31 & 37 & 29 & 27 & 1.1 & 150 & 33 & 5.8 & 87 & 2.19 & 5.06 & -0.17 & 2.29 \\
\hline Co & $\mathrm{mg} / \mathrm{kg}$ & 31 & 7.6 & 5.1 & 5.0 & 1.2 & 36 & 7.9 & 1.4 & 100 & 2.62 & 6.88 & -0.26 & 0.86 \\
\hline $\mathrm{Cr}$ & $\mathrm{mg} / \mathrm{kg}$ & 33 & 41 & 15 & 15 & 2.6 & 540 & 97 & 17 & 240 & 4.75 & 24.29 & 0.04 & 0.61 \\
\hline Cs & $\mathrm{mg} / \mathrm{kg}$ & 31 & 3.6 & 2.5 & 2.7 & 0.12 & 26 & 4.6 & 0.83 & 130 & 4.20 & 20.25 & 0.03 & 2.64 \\
\hline $\mathrm{Cu}$ & $\mathrm{mg} / \mathrm{kg}$ & 33 & 8.3 & 6.1 & 5.8 & 2.6 & 38 & 7.2 & 1.3 & 87 & 2.71 & 8.55 & 0.36 & -0.36 \\
\hline Dy & $\mathrm{mg} / \mathrm{kg}$ & 31 & 2.0 & 2.0 & 2.1 & 0.071 & 3.5 & 0.84 & 0.15 & 41 & -0.26 & 0.21 & -0.57 & 0.77 \\
\hline $\mathrm{Er}$ & $\mathrm{mg} / \mathrm{kg}$ & 31 & 1.0 & 1.0 & 1.1 & 0.038 & 1.8 & 0.41 & 0.073 & 39 & -0.44 & 0.59 & -0.55 & 0.79 \\
\hline $\mathrm{Eu}$ & $\mathrm{mg} / \mathrm{kg}$ & 31 & 0.67 & 0.63 & 0.60 & 0.024 & 1.8 & 0.38 & 0.068 & 56 & 1.01 & 1.74 & 0.01 & 1.01 \\
\hline $\mathrm{Fe}$ & $\%$ & 33 & 1.7 & 1.6 & 1.6 & 0.18 & 4.8 & 0.95 & 0.17 & 1700 & 1.66 & 4.08 & 0.42 & 2.22 \\
\hline Ga & $\mathrm{mg} / \mathrm{kg}$ & 33 & 12 & 12 & 11 & 0.24 & 31 & 5.3 & 0.92 & 45 & 1.05 & 5.61 & 0.75 & 4.95 \\
\hline Gd & $\mathrm{mg} / \mathrm{kg}$ & 31 & 2.7 & 2.6 & 2.7 & 0.10 & 5.9 & 1.3 & 0.24 & 49 & 0.48 & 0.43 & -0.18 & 0.76 \\
\hline Ge & $\mathrm{mg} / \mathrm{kg}$ & 31 & 0.53 & 0.43 & 0.46 & 0.039 & 1.7 & 0.45 & 0.081 & 86 & 1.05 & 0.48 & 0.30 & -0.87 \\
\hline $\mathrm{Hf}$ & $\mathrm{mg} / \mathrm{kg}$ & 31 & 0.58 & 0.42 & 0.38 & 0.034 & 2.3 & 0.55 & 0.10 & 97 & 2.10 & 3.68 & 0.17 & 1.48 \\
\hline Но & $\mathrm{mg} / \mathrm{kg}$ & 31 & 0.38 & 0.38 & 0.39 & 0.013 & 0.66 & 0.15 & 0.027 & 40 & -0.38 & 0.34 & -0.60 & 0.76 \\
\hline I & $\mu \mathrm{g} / \mathrm{kg}$ & 31 & 45 & 33 & 34 & 5.0 & 280 & 51 & 9.2 & 110 & 3.46 & 14.45 & 0.40 & 1.33 \\
\hline In & $\mu \mathrm{g} / \mathrm{kg}$ & 31 & 30 & 28 & 26 & 5.0 & 75 & 15 & 2.7 & 50 & 1.20 & 2.24 & 0.08 & 1.38 \\
\hline $\mathrm{K}$ & $\%$ & 33 & 1.3 & 1.4 & 1.3 & 0.034 & 2.4 & 0.56 & 0.098 & 980 & -0.35 & 0.21 & -0.23 & 0.04 \\
\hline $\mathrm{La}$ & $\mathrm{mg} / \mathrm{kg}$ & 31 & 19 & 16 & 16 & 0.55 & 51 & 13 & 2.4 & 69 & 0.57 & -0.66 & -0.42 & -0.20 \\
\hline $\mathrm{Li}$ & $\mathrm{mg} / \mathrm{kg}$ & 33 & 17 & 15 & 14 & 0.53 & 42 & 10 & 1.8 & 62 & 0.98 & 0.50 & -0.08 & 0.47 \\
\hline $\mathrm{Lu}$ & $\mathrm{mg} / \mathrm{kg}$ & 31 & 0.15 & 0.15 & 0.15 & 0.005 & 0.24 & 0.053 & 0.010 & 36 & -0.70 & 1.21 & -0.27 & 0.41 \\
\hline $\mathrm{Mg}$ & $\%$ & 33 & 1.1 & 0.47 & 0.48 & 0.17 & 8.8 & 1.8 & 0.32 & 3200 & 3.06 & 9.77 & 0.24 & -0.40 \\
\hline $\mathrm{Mn}$ & $\mathrm{mg} / \mathrm{kg}$ & 33 & 350 & 330 & 300 & 16 & 930 & 190 & 33 & 54 & 1.01 & 1.75 & 0.06 & 1.13 \\
\hline Mo & $\mathrm{mg} / \mathrm{kg}$ & 31 & 0.49 & 0.42 & 0.39 & 0.005 & 1.7 & 0.37 & 0.067 & 75 & 1.65 & 3.15 & -0.13 & 1.38 \\
\hline $\mathrm{Na}$ & $\%$ & 33 & 0.88 & 0.87 & 0.95 & 0.033 & 1.9 & 0.43 & 0.074 & 740 & -0.16 & 0.35 & -0.33 & 0.33 \\
\hline $\mathrm{Nb}$ & $\mathrm{mg} / \mathrm{kg}$ & 31 & 10 & 9.5 & 8.8 & 0.33 & 25 & 5.1 & 0.92 & 51 & 0.58 & 1.17 & -0.17 & 0.93 \\
\hline $\mathrm{Nd}$ & $\mathrm{mg} / \mathrm{kg}$ & 31 & 12 & 11 & 10 & 0.45 & 30 & 7.0 & 1.3 & 57 & 0.99 & 0.99 & -0.08 & 0.95 \\
\hline $\mathrm{Ni}$ & $\mathrm{mg} / \mathrm{kg}$ & 33 & 34 & 9.8 & 8.2 & 2.2 & 450 & 87 & 15 & 260 & 4.24 & 18.51 & 0.11 & 1.05 \\
\hline $\mathrm{P}$ & $\mathrm{mg} / \mathrm{kg}$ & 33 & 380 & 350 & 350 & 62 & 750 & 180 & 31 & 47 & 0.40 & -0.57 & -0.40 & 0.04 \\
\hline $\mathrm{Pb}$ & $\mathrm{mg} / \mathrm{kg}$ & 33 & 15 & 12 & 14 & 5.0 & 45 & 9.9 & 1.7 & 66 & 1.02 & 1.21 & -0.26 & -1.41 \\
\hline $\mathrm{Pd}$ & $\mathrm{mg} / \mathrm{kg}$ & 31 & 0.24 & 0.11 & 0.13 & 0.050 & 1.6 & 0.36 & 0.064 & 150 & 2.95 & 8.54 & 0.29 & -1.20 \\
\hline $\operatorname{Pr}$ & $\mathrm{mg} / \mathrm{kg}$ & 31 & 3.2 & 2.9 & 2.6 & 0.12 & 8.2 & 1.9 & 0.35 & 60 & 1.15 & 1.27 & -0.01 & 1.04 \\
\hline $\mathrm{Pt}$ & $\mathrm{mg} / \mathrm{kg}$ & 31 & 0.28 & 0.26 & 0.19 & 0.12 & 0.61 & 0.16 & 0.028 & 55 & 0.72 & -0.96 & 0.51 & -1.34 \\
\hline $\mathrm{Rb}$ & $\mathrm{mg} / \mathrm{kg}$ & 31 & 52 & 45 & 40 & 1.7 & 160 & 36 & 6.5 & 70 & 1.44 & 2.23 & -0.09 & 1.03 \\
\hline $\mathrm{Sb}$ & $\mathrm{mg} / \mathrm{kg}$ & 31 & 0.28 & 0.21 & 0.21 & 0.071 & 1.6 & 0.27 & 0.049 & 98 & 3.95 & 18.11 & 0.21 & 1.15 \\
\hline Sc & $\mathrm{mg} / \mathrm{kg}$ & 31 & 3.1 & 2.6 & 2.4 & 0.050 & 7.5 & 1.9 & 0.34 & 62 & 0.82 & -0.02 & -0.83 & 1.84 \\
\hline $\mathrm{Sm}$ & $\mathrm{mg} / \mathrm{kg}$ & 31 & 2.4 & 2.3 & 2.2 & 0.050 & 5.5 & 1.2 & 0.22 & 51 & 0.69 & 0.86 & -0.13 & 1.03 \\
\hline Sn & $\mathrm{mg} / \mathrm{kg}$ & 31 & 11 & 9.0 & 8.9 & 3.2 & 32 & 8.3 & 1.5 & 72 & 1.35 & 1.16 & 0.19 & -0.88 \\
\hline $\mathrm{Sr}$ & $\mathrm{mg} / \mathrm{kg}$ & 33 & 120 & 96 & 99 & 32 & 490 & 88 & 15 & 76 & 2.70 & 9.42 & 0.57 & 0.43 \\
\hline Тa & $\mathrm{mg} / \mathrm{kg}$ & 31 & 0.76 & 0.74 & 0.73 & 0.050 & 1.7 & 0.39 & 0.070 & 51 & 0.30 & 0.09 & -0.14 & 0.17 \\
\hline $\mathrm{Tb}$ & $\mathrm{mg} / \mathrm{kg}$ & 31 & 0.38 & 0.37 & 0.38 & 0.013 & 0.73 & 0.17 & 0.030 & 45 & 0.13 & 0.21 & -0.34 & 0.71 \\
\hline $\mathrm{Ti}$ & $\%$ & 31 & 0.16 & 0.15 & 0.15 & 0.013 & 0.27 & 0.062 & 0.011 & 110 & -0.63 & 0.36 & -1.00 & 1.12 \\
\hline $\mathrm{Tl}$ & $\mathrm{mg} / \mathrm{kg}$ & 31 & 0.47 & 0.44 & 0.43 & 0.050 & 1.2 & 0.25 & 0.046 & 55 & 1.25 & 2.25 & 0.28 & 1.46 \\
\hline $\mathrm{Tm}$ & $\mathrm{mg} / \mathrm{kg}$ & 31 & 0.15 & 0.15 & 0.15 & 0.005 & 0.26 & 0.056 & 0.010 & 38 & -0.56 & 0.90 & -0.55 & 0.88 \\
\hline V & $\mathrm{mg} / \mathrm{kg}$ & 33 & 40 & 36 & 33 & 4.9 & 110 & 23 & 4.1 & 59 & 1.34 & 1.98 & 0.31 & 0.60 \\
\hline $\mathrm{W}$ & $\mathrm{mg} / \mathrm{kg}$ & 31 & 1.4 & 0.93 & 0.88 & 0.29 & 8.2 & 1.6 & 0.29 & 110 & 3.04 & 10.84 & 0.51 & 0.02 \\
\hline $\mathrm{Y}$ & $\mathrm{mg} / \mathrm{kg}$ & 31 & 7.7 & 7.5 & 7.2 & 0.21 & 15 & 3.3 & 0.59 & 43 & -0.07 & 0.41 & -0.39 & 0.79 \\
\hline $\mathrm{Yb}$ & $\mathrm{mg} / \mathrm{kg}$ & 31 & 0.95 & 0.96 & 0.94 & 0.038 & 1.6 & 0.35 & 0.063 & 37 & -0.63 & 1.11 & -0.39 & 0.70 \\
\hline $\mathrm{Zn}$ & $\mathrm{mg} / \mathrm{kg}$ & 33 & 52 & 48 & 45 & 23 & 150 & 24 & 4.2 & 47 & 2.60 & 8.81 & 1.10 & 2.31 \\
\hline $\mathrm{Zr}$ & $\mathrm{mg} / \mathrm{kg}$ & 31 & 21 & 14 & 11 & 0.80 & 98 & 24 & 4.4 & 110 & 2.25 & 4.34 & -0.28 & 2.12 \\
\hline
\end{tabular}

$\mathrm{X}$ - arithmetic mean; BC - Box-Cox transformed values; Md - median; Min - minimum; Max - maximum; S - standard deviation; $\mathrm{S}_{\mathrm{x}}$ - standard deviation (standard error); CV - coefficient of variation; A - asymmetry; E - distribution. 
Table 3

Descriptive statistics for the content of the elements in subsoil $(20-30 \mathrm{~cm}), n=33$

\begin{tabular}{|c|c|c|c|c|c|c|c|c|c|c|c|c|c|c|}
\hline & Unit & $\mathrm{N}$ & $X$ & $X_{(\mathrm{BC})}$ & $\mathrm{Md}$ & Min & Max & S & $\mathrm{S}_{\mathrm{X}}$ & $\mathrm{CV}$ & A & $\mathrm{E}$ & $\mathrm{A}_{(\mathrm{BC})}$ & $\mathrm{E}_{(\mathrm{BC})}$ \\
\hline $\mathrm{Ag}$ & $\mathrm{mg} / \mathrm{kg}$ & 33 & 0.78 & 0.69 & 0.67 & 0.005 & 2.4 & 0.50 & 0.087 & 64 & 1.46 & 3.23 & -0.24 & 1.65 \\
\hline $\mathrm{Al}$ & $\%$ & 33 & 2.4 & 2.3 & 2.1 & 0.12 & 5.0 & 1.1 & 0.20 & 2000 & 0.64 & 0.35 & -0.09 & 0.81 \\
\hline As & $\mathrm{mg} / \mathrm{kg}$ & 33 & 22 & 13 & 13 & 3.2 & 170 & 30 & 5.3 & 140 & 4.37 & 21.56 & -0.09 & 0.70 \\
\hline $\mathrm{Ba}$ & $\mathrm{mg} / \mathrm{kg}$ & 33 & 810 & 740 & 780 & 77 & 2300 & 500 & 87 & 62 & 0.98 & 1.43 & 0.19 & 0.03 \\
\hline $\mathrm{Be}$ & $\mathrm{mg} / \mathrm{kg}$ & 33 & 2.1 & 2.0 & 2.1 & 0.005 & 4.1 & 0.97 & 0.17 & 46 & -0.23 & 0.53 & -0.86 & 1.24 \\
\hline $\mathrm{Bi}$ & $\mathrm{mg} / \mathrm{kg}$ & 31 & 0.24 & 0.17 & 0.17 & 0.067 & 1.8 & 0.30 & 0.054 & 130 & 5.05 & 27.00 & -0.15 & 1.39 \\
\hline $\mathrm{Br}$ & $\mathrm{mg} / \mathrm{kg}$ & 31 & 3.5 & 2.2 & 2.0 & 0.005 & 32 & 5.8 & 1.0 & 160 & 4.36 & 20.83 & 0.75 & 4.73 \\
\hline $\mathrm{Ca}$ & $\%$ & 33 & 3.3 & 1.2 & 1.1 & 0.25 & 18 & 5.3 & 0.92 & 9200 & 2.14 & 3.10 & 0.01 & 0.69 \\
\hline $\mathrm{Cd}$ & $\mu \mathrm{g} / \mathrm{kg}$ & 33 & 53 & 17 & 12 & 5.0 & 820 & 140 & 25 & 270 & 5.13 & 27.80 & 0.62 & 0.56 \\
\hline Ce & $\mathrm{mg} / \mathrm{kg}$ & 31 & 40 & 33 & 29 & 8.4 & 130 & 31 & 5.6 & 77 & 1.89 & 3.50 & 0.63 & 0.31 \\
\hline Co & $\mathrm{mg} / \mathrm{kg}$ & 31 & 8.8 & 5.8 & 5.9 & 1.9 & 47 & 11 & 1.9 & 120 & 3.13 & 9.43 & 0.42 & 1.12 \\
\hline $\mathrm{Cr}$ & $\mathrm{mg} / \mathrm{kg}$ & 33 & 48 & 14 & 13 & 1.8 & 690 & 130 & 22 & 260 & 4.54 & 21.83 & -0.08 & 0.98 \\
\hline Cs & $\mathrm{mg} / \mathrm{kg}$ & 31 & 3.4 & 2.5 & 2.5 & 0.15 & 22 & 3.9 & 0.71 & 110 & 3.66 & 16.00 & 0.10 & 2.47 \\
\hline $\mathrm{Cu}$ & $\mathrm{mg} / \mathrm{kg}$ & 33 & 7.5 & 5.1 & 4.8 & 1.6 & 26 & 6.6 & 1.2 & 88 & 1.65 & 1.69 & 0.06 & -0.37 \\
\hline Dy & $\mathrm{mg} / \mathrm{kg}$ & 31 & 2.2 & 2.2 & 2.0 & 0.64 & 5.0 & 0.95 & 0.17 & 43 & 0.68 & 0.93 & 0.47 & 0.50 \\
\hline Er & $\mathrm{mg} / \mathrm{kg}$ & 31 & 1.1 & 1.1 & 1.1 & 0.34 & 2.4 & 0.45 & 0.082 & 40 & 0.43 & 0.71 & 0.35 & 0.57 \\
\hline $\mathrm{Eu}$ & $\mathrm{mg} / \mathrm{kg}$ & 31 & 0.72 & 0.68 & 0.67 & 0.18 & 1.6 & 0.35 & 0.062 & 48 & 0.54 & -0.14 & 0.12 & -0.59 \\
\hline $\mathrm{Fe}$ & $\%$ & 33 & 1.8 & 1.7 & 1.7 & 0.029 & 5.0 & 0.97 & 0.17 & 1700 & 1.38 & 3.44 & -0.02 & 2.24 \\
\hline Ga & $\mathrm{mg} / \mathrm{kg}$ & 33 & 12 & 12 & 12 & 1.5 & 19 & 4.6 & 0.80 & 39 & -0.64 & 0.20 & -0.73 & 0.34 \\
\hline Gd & $\mathrm{mg} / \mathrm{kg}$ & 31 & 3.0 & 2.9 & 2.8 & 0.87 & 6.3 & 1.4 & 0.25 & 46 & 0.44 & -0.55 & 0.19 & -0.82 \\
\hline Ge & $\mathrm{mg} / \mathrm{kg}$ & 31 & 0.67 & 0.59 & 0.73 & 0.019 & 1.6 & 0.39 & 0.071 & 59 & 0.24 & -0.02 & -0.70 & 0.00 \\
\hline $\mathrm{Hf}$ & $\mathrm{mg} / \mathrm{kg}$ & 31 & 0.56 & 0.41 & 0.40 & 0.028 & 2.5 & 0.55 & 0.099 & 97 & 2.25 & 5.32 & -0.08 & 1.34 \\
\hline Но & $\mathrm{mg} / \mathrm{kg}$ & 31 & 0.42 & 0.41 & 0.40 & 0.12 & 0.92 & 0.17 & 0.031 & 42 & 0.61 & 1.00 & 0.44 & 0.68 \\
\hline I & $\mu \mathrm{g} / \mathrm{kg}$ & 31 & 56 & 41 & 46 & 5.0 & 240 & 50 & 8.9 & 89 & 2.19 & 6.10 & -0.37 & 0.26 \\
\hline In & $\mu \mathrm{g} / \mathrm{kg}$ & 31 & 27 & 26 & 26 & 5.0 & 54 & 10 & 1.8 & 38 & 1.08 & 2.18 & 0.00 & 2.55 \\
\hline K & $\%$ & 33 & 1.2 & 1.2 & 1.3 & 0.022 & 2.2 & 0.51 & 0.089 & 890 & -0.62 & 0.13 & -0.52 & 0.00 \\
\hline $\mathrm{La}$ & $\mathrm{mg} / \mathrm{kg}$ & 31 & 19 & 16 & 12 & 3.1 & 64 & 15 & 2.6 & 77 & 1.40 & 1.63 & 0.53 & -0.46 \\
\hline $\mathrm{Li}$ & $\mathrm{mg} / \mathrm{kg}$ & 33 & 17 & 15 & 13 & 0.52 & 43 & 11 & 1.9 & 67 & 1.02 & 0.37 & 0.08 & 0.10 \\
\hline $\mathrm{Lu}$ & $\mathrm{mg} / \mathrm{kg}$ & 31 & 0.16 & 0.16 & 0.16 & 0.049 & 0.27 & 0.055 & 0.010 & 36 & -0.37 & -0.45 & -0.18 & -0.43 \\
\hline $\mathrm{Mg}$ & $\%$ & 33 & 1.1 & 0.46 & 0.43 & 0.15 & 7.4 & 1.8 & 0.31 & 3100 & 2.70 & 6.67 & 0.11 & -0.45 \\
\hline $\mathrm{Mn}$ & $\mathrm{mg} / \mathrm{kg}$ & 33 & 320 & 300 & 280 & 8.1 & 730 & 170 & 30 & 55 & 0.79 & 0.31 & 0.03 & 0.50 \\
\hline Mo & $\mathrm{mg} / \mathrm{kg}$ & 31 & 0.53 & 0.48 & 0.38 & 0.19 & 1.7 & 0.34 & 0.062 & 65 & 2.04 & 4.49 & 1.29 & 1.65 \\
\hline $\mathrm{Na}$ & $\%$ & 33 & 0.81 & 0.80 & 0.91 & 0.042 & 1.9 & 0.43 & 0.075 & 750 & -0.04 & 0.14 & -0.19 & -0.01 \\
\hline $\mathrm{Nb}$ & $\mathrm{mg} / \mathrm{kg}$ & 31 & 12 & 11 & 11 & 0.54 & 31 & 6.2 & 1.1 & 54 & 0.88 & 2.22 & 0.07 & 1.19 \\
\hline $\mathrm{Nd}$ & $\mathrm{mg} / \mathrm{kg}$ & 31 & 14 & 13 & 12 & 3.7 & 28 & 6.8 & 1.2 & 50 & 0.55 & -0.90 & 0.24 & -1.09 \\
\hline $\mathrm{Ni}$ & $\mathrm{mg} / \mathrm{kg}$ & 33 & 36 & 8.9 & 8.8 & 2.7 & 530 & 99 & 17 & 280 & 4.50 & 21.33 & 0.05 & -0.17 \\
\hline $\mathrm{P}$ & $\mathrm{mg} / \mathrm{kg}$ & 33 & 390 & 350 & 350 & 74 & 1100 & 230 & 40 & 59 & 1.39 & 2.45 & 0.28 & 0.18 \\
\hline $\mathrm{Pb}$ & $\mathrm{mg} / \mathrm{kg}$ & 33 & 11 & 8.5 & 5.0 & 5.0 & 31 & 7.1 & 1.2 & 66 & 1.03 & 0.26 & 0.36 & -1.61 \\
\hline $\mathrm{Pd}$ & $\mathrm{mg} / \mathrm{kg}$ & 31 & 0.25 & 0.12 & 0.14 & 0.050 & 1.4 & 0.33 & 0.060 & 130 & 2.34 & 5.13 & 0.09 & -1.41 \\
\hline $\operatorname{Pr}$ & $\mathrm{mg} / \mathrm{kg}$ & 31 & 3.6 & 3.3 & 3.1 & 0.98 & 7.1 & 1.8 & 0.33 & 51 & 0.54 & -0.92 & 0.21 & -1.07 \\
\hline $\mathrm{Pt}$ & $\mathrm{mg} / \mathrm{kg}$ & 31 & 0.25 & 0.22 & 0.21 & 0.005 & 0.81 & 0.17 & 0.030 & 66 & 1.70 & 3.54 & -0.03 & 2.04 \\
\hline $\mathrm{Rb}$ & $\mathrm{mg} / \mathrm{kg}$ & 31 & 47 & 43 & 45 & 10 & 160 & 29 & 5.2 & 62 & 1.93 & 6.58 & 0.32 & 0.99 \\
\hline $\mathrm{Sb}$ & $\mathrm{mg} / \mathrm{kg}$ & 31 & 0.23 & 0.18 & 0.18 & 0.054 & 0.82 & 0.17 & 0.030 & 74 & 2.67 & 7.42 & -0.34 & 2.15 \\
\hline Sc & $\mathrm{mg} / \mathrm{kg}$ & 31 & 3.7 & 3.2 & 2.9 & 0.88 & 17 & 3.2 & 0.57 & 86 & 2.66 & 8.60 & 1.16 & 1.97 \\
\hline $\mathrm{Sm}$ & $\mathrm{mg} / \mathrm{kg}$ & 31 & 2.7 & 2.6 & 2.4 & 0.71 & 5.2 & 1.3 & 0.23 & 47 & 0.47 & -0.82 & 0.23 & -0.97 \\
\hline Sn & $\mathrm{mg} / \mathrm{kg}$ & 31 & 21 & 15 & 14 & 2.0 & 100 & 19 & 3.4 & 91 & 2.70 & 9.99 & -0.39 & 0.59 \\
\hline $\mathrm{Sr}$ & $\mathrm{mg} / \mathrm{kg}$ & 33 & 120 & 93 & 99 & 11 & 520 & 95 & 17 & 81 & 2.70 & 9.48 & -0.34 & 2.21 \\
\hline Тa & $\mathrm{mg} / \mathrm{kg}$ & 31 & 0.83 & 0.79 & 0.86 & 0.050 & 1.7 & 0.45 & 0.080 & 54 & 0.12 & -0.77 & -0.20 & -0.59 \\
\hline $\mathrm{Tb}$ & $\mathrm{mg} / \mathrm{kg}$ & 31 & 0.41 & 0.40 & 0.39 & 0.11 & 0.89 & 0.18 & 0.033 & 44 & 0.55 & 0.09 & 0.31 & -0.24 \\
\hline $\mathrm{Ti}$ & $\%$ & 31 & 0.18 & 0.18 & 0.17 & 0.024 & 0.42 & 0.086 & 0.015 & 160 & 0.93 & 1.55 & 0.50 & 1.04 \\
\hline $\mathrm{Tl}$ & $\mathrm{mg} / \mathrm{kg}$ & 31 & 0.44 & 0.42 & 0.42 & 0.050 & 1.2 & 0.23 & 0.042 & 53 & 0.81 & 2.50 & -0.18 & 0.92 \\
\hline $\mathrm{Tm}$ & $\mathrm{mg} / \mathrm{kg}$ & 31 & 0.16 & 0.16 & 0.16 & 0.049 & 0.33 & 0.062 & 0.011 & 39 & 0.29 & 0.57 & 0.30 & 0.58 \\
\hline $\mathrm{V}$ & $\mathrm{mg} / \mathrm{kg}$ & 33 & 44 & 39 & 41 & 1.4 & 110 & 27 & 4.7 & 62 & 0.99 & 0.63 & -0.16 & 0.55 \\
\hline W & $\mathrm{mg} / \mathrm{kg}$ & 31 & 1.2 & 0.82 & 0.77 & 0.11 & 4.7 & 1.1 & 0.20 & 92 & 1.83 & 2.99 & -0.37 & 0.78 \\
\hline $\mathrm{Y}$ & $\mathrm{mg} / \mathrm{kg}$ & 31 & 8.5 & 8.4 & 8.4 & 2.1 & 20 & 3.5 & 0.63 & 41 & 0.90 & 3.31 & 0.55 & 2.52 \\
\hline $\mathrm{Yb}$ & $\mathrm{mg} / \mathrm{kg}$ & 31 & 1.0 & 1.0 & 1.1 & 0.33 & 1.9 & 0.38 & 0.067 & 37 & -0.08 & 0.06 & 0.07 & 0.21 \\
\hline $\mathrm{Zn}$ & $\mathrm{mg} / \mathrm{kg}$ & 33 & 48 & 42 & 42 & 7.8 & 180 & 30 & 5.2 & 62 & 2.85 & 11.29 & -0.15 & 3.04 \\
\hline $\mathrm{Zr}$ & $\mathrm{mg} / \mathrm{kg}$ & 31 & 22 & 14 & 13 & 2.3 & 96 & 24 & 4.3 & 110 & 2.08 & 3.39 & 0.35 & 0.24 \\
\hline
\end{tabular}

$\mathrm{X}$ - arithmetic mean; BC - Box-Cox transformed values; Md - median; Min - minimum; Max - maximum; S - standard deviation; $\mathrm{S}_{\mathrm{x}}$ - standard deviation (standard error); CV - coefficient of variation; A - asymmetry; E - distribution. 
Table 4

Ratio of the average contents (topsoil vs. subsoil)

\begin{tabular}{|c|c|c|c|c|c|c|c|c|c|c|}
\hline & Unit & Topsoil & Subsoil & $\mathrm{FO}(\mathrm{T} / \mathrm{B})$ & $\mathrm{T}$ (test) & Sign & F(ratio) & Sign & $\mathrm{R}(\mathrm{T} / \mathrm{B})$ & Sign \\
\hline $\mathrm{Ag}$ & $\mathrm{mg} / \mathrm{kg}$ & 0.64 & 0.69 & 0.93 & -0.41 & NS & 1.12 & NS & 0.28 & NS \\
\hline $\mathrm{Al}$ & $\%$ & 2.3 & 2.3 & 1.01 & 0.09 & NS & 1.08 & NS & 0.75 & $*$ \\
\hline As & $\mathrm{mg} / \mathrm{kg}$ & 13 & 13 & 0.94 & -0.35 & NS & 1.13 & NS & 0.24 & NS \\
\hline $\mathrm{Ba}$ & $\mathrm{mg} / \mathrm{kg}$ & 760 & 740 & 1.02 & 0.13 & NS & 1.18 & NS & 0.82 & $*$ \\
\hline $\mathrm{Be}$ & $\mathrm{mg} / \mathrm{kg}$ & 2 & 2 & 1.00 & -0.03 & NS & 1.32 & NS & 0.63 & * \\
\hline $\mathrm{Bi}$ & $\mathrm{mg} / \mathrm{kg}$ & 0.18 & 0.17 & 1.05 & 0.40 & NS & 1.09 & NS & 0.79 & * \\
\hline $\mathrm{Br}$ & $\mathrm{mg} / \mathrm{kg}$ & 1.5 & 2.2 & 0.66 & -1.33 & NS & 1.07 & NS & 0.15 & NS \\
\hline $\mathrm{Ca}$ & $\%$ & 1.3 & 1.2 & 1.05 & 0.22 & NS & 1.13 & NS & 0.93 & $*$ \\
\hline $\mathrm{Cd}$ & $\mu \mathrm{g} / \mathrm{kg}$ & 33 & 17 & 1.93 & 2.66 & $*$ & 1.42 & $*$ & 0.45 & * \\
\hline $\mathrm{Ce}$ & $\mathrm{mg} / \mathrm{kg}$ & 29 & 33 & 0.87 & -0.69 & NS & 1.39 & NS & 0.73 & $*$ \\
\hline Co & $\mathrm{mg} / \mathrm{kg}$ & 5.1 & 5.8 & 0.87 & -0.79 & NS & 1.38 & NS & 0.84 & * \\
\hline $\mathrm{Cr}$ & $\mathrm{mg} / \mathrm{kg}$ & 15 & 14 & 1.08 & 0.30 & NS & 1.20 & NS & 0.88 & * \\
\hline Cs & $\mathrm{mg} / \mathrm{kg}$ & 2.5 & 2.5 & 1.00 & -0.01 & NS & 1.15 & NS & 0.77 & $*$ \\
\hline $\mathrm{Cu}$ & $\mathrm{mg} / \mathrm{kg}$ & 6.1 & 5.1 & 1.20 & 1.19 & NS & 1.65 & NS & 0.82 & $*$ \\
\hline Dy & $\mathrm{mg} / \mathrm{kg}$ & 2 & 2.2 & 0.91 & -0.82 & NS & 1.14 & NS & 0.57 & * \\
\hline Er & $\mathrm{mg} / \mathrm{kg}$ & 1 & 1.1 & 0.93 & -0.76 & NS & 1.21 & NS & 0.57 & * \\
\hline $\mathrm{Eu}$ & $\mathrm{mg} / \mathrm{kg}$ & 0.63 & 0.68 & 0.92 & -0.59 & NS & 1.30 & NS & 0.60 & * \\
\hline $\mathrm{Fe}$ & $\%$ & 1.6 & 1.7 & 0.96 & -0.27 & NS & 1.10 & NS & 0.93 & $*$ \\
\hline $\mathrm{Ga}$ & $\mathrm{mg} / \mathrm{kg}$ & 12 & 12 & 0.97 & -0.28 & NS & 1.27 & NS & 0.53 & $*$ \\
\hline Gd & $\mathrm{mg} / \mathrm{kg}$ & 2.6 & 2.9 & 0.89 & -0.90 & NS & 1.06 & NS & 0.58 & $*$ \\
\hline $\mathrm{Ge}$ & $\mathrm{mg} / \mathrm{kg}$ & 0.43 & 0.59 & 0.72 & -1.55 & NS & 1.26 & NS & 0.00 & NS \\
\hline $\mathrm{Hf}$ & $\mathrm{mg} / \mathrm{kg}$ & 0.42 & 0.41 & 1.04 & 0.17 & NS & 1.11 & NS & 0.82 & $*$ \\
\hline Но & $\mathrm{mg} / \mathrm{kg}$ & 0.38 & 0.41 & 0.92 & -0.80 & NS & 1.18 & NS & 0.57 & * \\
\hline I & $\mu \mathrm{g} / \mathrm{kg}$ & 33 & 41 & 0.79 & -0.97 & NS & 1.18 & NS & 0.42 & $*$ \\
\hline In & $\mu \mathrm{g} / \mathrm{kg}$ & 28 & 26 & 1.07 & 0.56 & NS & 1.88 & NS & 0.49 & $*$ \\
\hline $\mathrm{K}$ & $\%$ & 1.4 & 1.2 & 1.13 & 1.19 & NS & 1.23 & NS & 0.52 & $*$ \\
\hline $\mathrm{La}$ & $\mathrm{mg} / \mathrm{kg}$ & 16 & 16 & 0.99 & -0.04 & NS & 1.24 & NS & 0.74 & * \\
\hline $\mathrm{Li}$ & $\mathrm{mg} / \mathrm{kg}$ & 15 & 15 & 1.01 & 0.08 & NS & 1.10 & NS & 0.93 & * \\
\hline $\mathrm{Lu}$ & $\mathrm{mg} / \mathrm{kg}$ & 0.15 & 0.16 & 0.96 & -0.53 & NS & 1.17 & NS & 0.56 & * \\
\hline $\mathrm{Mg}$ & $\%$ & 0.47 & 0.46 & 1.03 & 0.14 & NS & 1.13 & NS & 0.91 & $*$ \\
\hline $\mathrm{Mn}$ & $\mathrm{mg} / \mathrm{kg}$ & 330 & 300 & 1.11 & 0.72 & NS & 1.08 & NS & 0.92 & $*$ \\
\hline Mo & $\mathrm{mg} / \mathrm{kg}$ & 0.42 & 0.48 & 0.87 & -0.78 & NS & 1.81 & NS & 0.09 & NS \\
\hline $\mathrm{Na}$ & $\%$ & 0.87 & 0.8 & 1.09 & 0.63 & NS & 1.04 & NS & 0.61 & $*$ \\
\hline $\mathrm{Nb}$ & $\mathrm{mg} / \mathrm{kg}$ & 9.5 & 11 & 0.87 & -1.01 & NS & 1.30 & NS & 0.52 & $*$ \\
\hline $\mathrm{Nd}$ & $\mathrm{mg} / \mathrm{kg}$ & 11 & 13 & 0.88 & -0.86 & NS & 1.22 & NS & 0.65 & $*$ \\
\hline $\mathrm{Ni}$ & $\mathrm{mg} / \mathrm{kg}$ & 9.8 & 8.9 & 1.10 & 0.47 & NS & 1.29 & NS & 0.75 & * \\
\hline $\mathrm{P}$ & $\mathrm{mg} / \mathrm{kg}$ & 350 & 350 & 1.02 & 0.13 & NS & 1.27 & NS & 0.85 & $*$ \\
\hline $\mathrm{Pb}$ & $\mathrm{mg} / \mathrm{kg}$ & 12 & 8.5 & 1.36 & 1.89 & NS & 1.17 & NS & 0.63 & $*$ \\
\hline $\mathrm{Pd}$ & $\mathrm{mg} / \mathrm{kg}$ & 0.11 & 0.12 & 0.91 & -0.43 & NS & 1.05 & NS & 0.65 & * \\
\hline $\mathrm{Pr}$ & $\mathrm{mg} / \mathrm{kg}$ & 2.9 & 3.3 & 0.88 & -0.85 & NS & 1.29 & NS & 0.66 & * \\
\hline $\mathrm{Pt}$ & $\mathrm{mg} / \mathrm{kg}$ & 0.26 & 0.22 & 1.17 & 0.97 & NS & 1.37 & NS & 0.54 & * \\
\hline $\mathrm{Rb}$ & $\mathrm{mg} / \mathrm{kg}$ & 45 & 43 & 1.05 & 0.31 & NS & 1.57 & NS & 0.48 & * \\
\hline $\mathrm{Sb}$ & $\mathrm{mg} / \mathrm{kg}$ & 0.21 & 0.18 & 1.15 & 1.02 & NS & 1.04 & NS & 0.86 & $*$ \\
\hline Sc & $\mathrm{mg} / \mathrm{kg}$ & 2.6 & 3.2 & 0.83 & -0.99 & NS & 1.01 & NS & 0.53 & * \\
\hline $\mathrm{Sm}$ & $\mathrm{mg} / \mathrm{kg}$ & 2.3 & 2.6 & 0.89 & -0.87 & NS & 1.13 & NS & 0.63 & $*$ \\
\hline Sn & $\mathrm{mg} / \mathrm{kg}$ & 9 & 15 & 0.60 & -2.69 & $*$ & 1.39 & NS & -0.09 & NS \\
\hline $\mathrm{Sr}$ & $\mathrm{mg} / \mathrm{kg}$ & 96 & 93 & 1.03 & 0.16 & NS & 1.38 & NS & 0.92 & $*$ \\
\hline Та & $\mathrm{mg} / \mathrm{kg}$ & 0.74 & 0.79 & 0.93 & -0.52 & NS & 1.28 & NS & 0.59 & * \\
\hline $\mathrm{Tb}$ & $\mathrm{mg} / \mathrm{kg}$ & 0.37 & 0.4 & 0.90 & -0.85 & NS & 1.03 & NS & 0.56 & $*$ \\
\hline $\mathrm{Ti}$ & $\%$ & 0.15 & 0.18 & 0.88 & -1.13 & NS & 1.57 & NS & 0.61 & * \\
\hline $\mathrm{Tl}$ & $\mathrm{mg} / \mathrm{kg}$ & 0.44 & 0.42 & 1.06 & 0.39 & NS & 1.04 & NS & 0.79 & * \\
\hline $\mathrm{Tm}$ & $\mathrm{mg} / \mathrm{kg}$ & 0.15 & 0.16 & 0.94 & -0.68 & NS & 1.23 & NS & 0.54 & * \\
\hline $\mathrm{V}$ & $\mathrm{mg} / \mathrm{kg}$ & 36 & 39 & 0.94 & -0.40 & NS & 1.39 & NS & 0.94 & * \\
\hline W & $\mathrm{mg} / \mathrm{kg}$ & 0.93 & 0.82 & 1.13 & 0.60 & NS & 1.20 & NS & 0.77 & $*$ \\
\hline $\mathrm{Y}$ & $\mathrm{mg} / \mathrm{kg}$ & 7.5 & 8.4 & 0.90 & -0.96 & NS & 1.01 & NS & 0.46 & $*$ \\
\hline $\mathrm{Yb}$ & $\mathrm{mg} / \mathrm{kg}$ & 0.96 & 1 & 0.94 & -0.66 & NS & 1.23 & NS & 0.52 & * \\
\hline $\mathrm{Zn}$ & $\mathrm{mg} / \mathrm{kg}$ & 48 & 42 & 1.14 & 1.14 & NS & 2.01 & NS & 0.60 & * \\
\hline $\mathrm{Zr}$ & $\mathrm{mg} / \mathrm{kg}$ & 14 & 14 & 0.96 & -0.18 & NS & 1.15 & NS & 0.79 & * \\
\hline
\end{tabular}

FO (T/B) - Enrichment Factor: ratio of the content in topsoil (T) and bottom soil (B); Sign - significant at p < 0.01; R (T/B) - ration between the results for top and bottom soil; NS - difference not significant. 
Table 5

Comparison of the median, minimum and maximum values of the content of the analyzed elements in topsoil from the Mariovo region with soil from North Macedonia and Europe

\begin{tabular}{|c|c|c|c|c|c|c|c|c|c|}
\hline \multirow[t]{2}{*}{ Element } & \multirow[t]{2}{*}{ Unit } & \multicolumn{2}{|c|}{ Dutchlist } & \multicolumn{2}{|c|}{$\begin{array}{l}\text { Mariovo Region } \\
\text { (this work) }\end{array}$} & \multicolumn{2}{|c|}{$\begin{array}{c}\text { North Macedonia } \\
\text { (Stafilov and Šajn, 2019) }\end{array}$} & \multicolumn{2}{|c|}{$\begin{array}{c}\text { Europe } \\
\text { (Salminen et al., 2005) }\end{array}$} \\
\hline & & Target & Action & Md & Min-Max & $\mathrm{Md}$ & Min-Max & $\mathrm{Md}$ & Min-Max \\
\hline $\mathrm{Ag}$ & $\mathrm{mg} / \mathrm{kg}$ & & & 0.51 & $0.12-1.9$ & - & - & 0.27 & $0.01-3.15$ \\
\hline $\mathrm{Al}$ & $\%$ & & & 2.2 & $0.19-5.6$ & 1.3 & $0.05-35$ & 5.8 & $0.70-14.1$ \\
\hline As & $\mathrm{mg} / \mathrm{kg}$ & 29 & 55 & 13 & 3.7-100 & 10 & $1.0-720$ & 12 & $0.32-562$ \\
\hline $\mathrm{Ba}$ & $\mathrm{mg} / \mathrm{kg}$ & 200 & 625 & 740 & $52-2000$ & 430 & 6-2900 & 375 & $30-1870$ \\
\hline $\mathrm{Be}$ & $\mathrm{mg} / \mathrm{kg}$ & & & 2.0 & $0.005-6.7$ & 2.0 & $<1.0-8.0$ & $<2.0$ & $<2.0-18.7$ \\
\hline $\mathrm{Bi}$ & $\mathrm{mg} / \mathrm{kg}$ & & & 0.17 & $0.070-1.4$ & 0.30 & $<0.10-15$ & $<0.5$ & $<0.5-9.57$ \\
\hline $\mathrm{Br}$ & $\mathrm{mg} / \mathrm{kg}$ & & & 1.5 & 0.005-18 & - & - & - & - \\
\hline $\mathrm{Ca}$ & $\%$ & & & 1.3 & $0.29-17$ & 1.3 & $0.05-35$ & 0.66 & $0.019-34.3$ \\
\hline $\mathrm{Cd}$ & $\mathrm{mg} / \mathrm{kg}$ & 0.8 & 12 & 0.013 & $0.005-0.73$ & 0.30 & $0.01-110$ & 0.92 & 0.03-14 \\
\hline $\mathrm{Ce}$ & $\mathrm{mg} / \mathrm{kg}$ & & & 27 & 1.1-150 & 56 & $1.0-180$ & 48.2 & $1.04-379$ \\
\hline Co & $\mathrm{mg} / \mathrm{kg}$ & 20 & 240 & 5.0 & $1.2-36$ & 17 & $0.50-150$ & 8.0 & $<1.0-191$ \\
\hline $\mathrm{Cr}$ & $\mathrm{mg} / \mathrm{kg}$ & 100 & 380 & 15 & $2.6-540$ & 88 & $5.0-2700$ & 60 & $<3-6230$ \\
\hline Cs & $\mathrm{mg} / \mathrm{kg}$ & & & 2.7 & $0.12-26$ & - & - & 2.71 & $<0.5-69$ \\
\hline $\mathrm{Cu}$ & $\mathrm{mg} / \mathrm{kg}$ & 36 & 190 & 5.8 & $2.6-38$ & 28 & $1.6-270$ & 13 & $0.81-256$ \\
\hline Dy & $\mathrm{mg} / \mathrm{kg}$ & & & 2.1 & $0.071-3.5$ & - & - & 3.42 & $0.18-45$ \\
\hline $\mathrm{Er}$ & $\mathrm{mg} / \mathrm{kg}$ & & & 1.1 & $0.038-1.8$ & - & - & 1.98 & $0.12-26$ \\
\hline $\mathrm{Eu}$ & $\mathrm{mg} / \mathrm{kg}$ & & & 0.60 & $0.024-1.8$ & - & - & 0.77 & $0.05-7.0$ \\
\hline $\mathrm{Fe}$ & $\%$ & & & 1.6 & $0.18-4.8$ & 3.5 & $0.03-12$ & 1.34 & 0.049-10.6 \\
\hline Ga & $\mathrm{mg} / \mathrm{kg}$ & & & 11 & $0.24-31$ & - & - & 13.5 & $0.54-34$ \\
\hline Gd & $\mathrm{mg} / \mathrm{kg}$ & & & 2.7 & $0.10-5.9$ & - & - & 3.85 & $0.20-36$ \\
\hline Ge & $\mathrm{mg} / \mathrm{kg}$ & & & 0.46 & $0.039-1.7$ & - & - & - & - \\
\hline $\mathrm{Hf}$ & $\mathrm{mg} / \mathrm{kg}$ & & & 0.38 & $0.034-2.3$ & 1.0 & $<0.10-6.6$ & 5.55 & $<0.2-21$ \\
\hline Ho & $\mathrm{mg} / \mathrm{kg}$ & & & 0.39 & $0.013-0.66$ & - & - & 0.72 & $0.03-9.2$ \\
\hline I & $\mathrm{mg} / \mathrm{kg}$ & & & 0.034 & $0.005-0.28$ & - & - & 3.94 & $0.002-70.8$ \\
\hline In & $\mathrm{mg} / \mathrm{kg}$ & & & 0.026 & $0.005-0.075$ & - & - & 0.05 & $0.01-0.41$ \\
\hline K & $\%$ & & & 1.3 & $0.034-2.4$ & 1.9 & $0.02-5.3$ & 1.59 & $0.022-5.1$ \\
\hline $\mathrm{La}$ & $\mathrm{mg} / \mathrm{kg}$ & & & 16 & $0.53-51$ & 25 & $0.60-88$ & 23 & $1.10-143$ \\
\hline $\mathrm{Li}$ & $\mathrm{mg} / \mathrm{kg}$ & & & 14 & $0.53-42$ & 26 & $1.8-210$ & - & - \\
\hline $\mathrm{Lu}$ & $\mathrm{mg} / \mathrm{kg}$ & & & 0.15 & $0.005-0.24$ & - & - & 0.30 & $<0.02-3.21$ \\
\hline $\mathrm{Mg}$ & $\%$ & & & 0.48 & $0.17-8.8$ & 0.94 & $0.12-13$ & 0.47 & $<0.006-15$ \\
\hline Mn & $\mathrm{mg} / \mathrm{kg}$ & & & 300 & 16-930 & 900 & 17-10000 & 510 & $31-6070$ \\
\hline Mo & $\mathrm{mg} / \mathrm{kg}$ & 10 & 200 & 0.39 & $0.005-1.7$ & 0.90 & $<0.10-51$ & 0.61 & $<0.10-21$ \\
\hline $\mathrm{Na}$ & $\%$ & & & 0.95 & $0.033-1.9$ & 1.3 & $0.007-3.7$ & 0.6 & $0.03-3.34$ \\
\hline $\mathrm{Nb}$ & $\mathrm{mg} / \mathrm{kg}$ & & & 8.8 & $0.33-25$ & 11 & $0.30-2000$ & 9.68 & $0.45-134$ \\
\hline Nd & $\mathrm{mg} / \mathrm{kg}$ & & & 10 & $0.45-30$ & - & - & 21 & $1.14-132$ \\
\hline $\mathrm{Ni}$ & $\mathrm{mg} / \mathrm{kg}$ & 35 & 210 & 8.2 & $2.2-450$ & 46 & $2.1-2500$ & 18 & $<2-2690$ \\
\hline $\mathrm{P}$ & $\mathrm{mg} / \mathrm{kg}$ & & & 350 & $62-750$ & 620 & $110-3900$ & 960 & 82-9900 \\
\hline $\mathrm{Pb}$ & $\mathrm{mg} / \mathrm{kg}$ & 85 & 530 & 15 & $5.0-45$ & 32 & $1.2-10000$ & 23 & 5.3-970 \\
\hline Pd & $\mathrm{mg} / \mathrm{kg}$ & & & 0.13 & $0.050-1.6$ & - & - & - & - \\
\hline $\operatorname{Pr}$ & $\mathrm{mg} / \mathrm{kg}$ & & & 2.6 & $0.12-8.2$ & - & - & 5.6 & $0.29-31.6$ \\
\hline $\mathrm{Pt}$ & $\mathrm{mg} / \mathrm{kg}$ & & & 0.19 & $0.12-0.61$ & - & - & - & - \\
\hline $\mathrm{Rb}$ & $\mathrm{mg} / \mathrm{kg}$ & & & 40 & $1.7-160$ & 86 & $0.70-390$ & 80 & $2-390$ \\
\hline $\mathrm{Sb}$ & $\mathrm{mg} / \mathrm{kg}$ & & & 0.21 & $0.071-1.6$ & 0.80 & $<0.10-630$ & 0.60 & $0.02-31$ \\
\hline Sc & $\mathrm{mg} / \mathrm{kg}$ & & & 2.4 & $0.050-7.5$ & 12 & $<1.0-39$ & 8.21 & $<0.50-54$ \\
\hline $\mathrm{Sm}$ & $\mathrm{mg} / \mathrm{kg}$ & & & 2.2 & $0.050-5.5$ & - & - & 3.96 & $0.23-30$ \\
\hline Sn & $\mathrm{mg} / \mathrm{kg}$ & & & 8.9 & 3.2-32 & 2.6 & $<0.10-680$ & 3.00 & $<2.0-106$ \\
\hline $\mathrm{Sr}$ & $\mathrm{mg} / \mathrm{kg}$ & & & 99 & $32-490$ & 140 & 21-1400 & 89 & 8-3120 \\
\hline Тa & $\mathrm{mg} / \mathrm{kg}$ & & & 0.73 & $0.050-1.7$ & 0.70 & $<0.10-30$ & 0.68 & $<0.05-6.8$ \\
\hline $\mathrm{Tb}$ & $\mathrm{mg} / \mathrm{kg}$ & & & 0.38 & $0.013-0.73$ & - & - & 0.60 & $0.03-7.0$ \\
\hline $\mathrm{Ti}$ & $\%$ & & & 0.15 & $0.013-0.27$ & 0.34 & $0.004-1.2$ & 0.34 & $0.012-3.27$ \\
\hline $\mathrm{Tl}$ & $\mathrm{mg} / \mathrm{kg}$ & & & 0.43 & $0.050-1.2$ & 0.70 & $<0.50-16$ & 0.66 & $0.05-24$ \\
\hline $\mathrm{Tm}$ & $\mathrm{mg} / \mathrm{kg}$ & & & 0.15 & $0.005-0.26$ & - & - & 0.30 & $0.05-4.03$ \\
\hline V & $\mathrm{mg} / \mathrm{kg}$ & & & 33 & 4.9-110 & 89 & $1.0-470$ & 60 & $2.7-537$ \\
\hline W & $\mathrm{mg} / \mathrm{kg}$ & & & 0.88 & $0.29-8.2$ & 1.3 & $0.20-18$ & $<5.0$ & $<5.0-14$ \\
\hline $\mathrm{Y}$ & $\mathrm{mg} / \mathrm{kg}$ & & & 7.2 & $0.21-15$ & 18 & $0.30-110$ & 21 & $<3.0-267$ \\
\hline $\mathrm{Yb}$ & $\mathrm{mg} / \mathrm{kg}$ & & & 0.94 & $0.038-1.6$ & - & - & 1.99 & $0.09-25$ \\
\hline $\mathrm{Zn}$ & $\mathrm{mg} / \mathrm{kg}$ & 140 & 720 & 45 & 23-150 & 83 & $8.0-10000$ & 52 & $<3-2900$ \\
\hline $\mathrm{Zr}$ & $\mathrm{mg} / \mathrm{kg}$ & & & 11 & $0.80-98$ & 35 & $0.80-210$ & 231 & $5.0-1060$ \\
\hline
\end{tabular}

Md - median; Min - minimum; Max - Maximum 


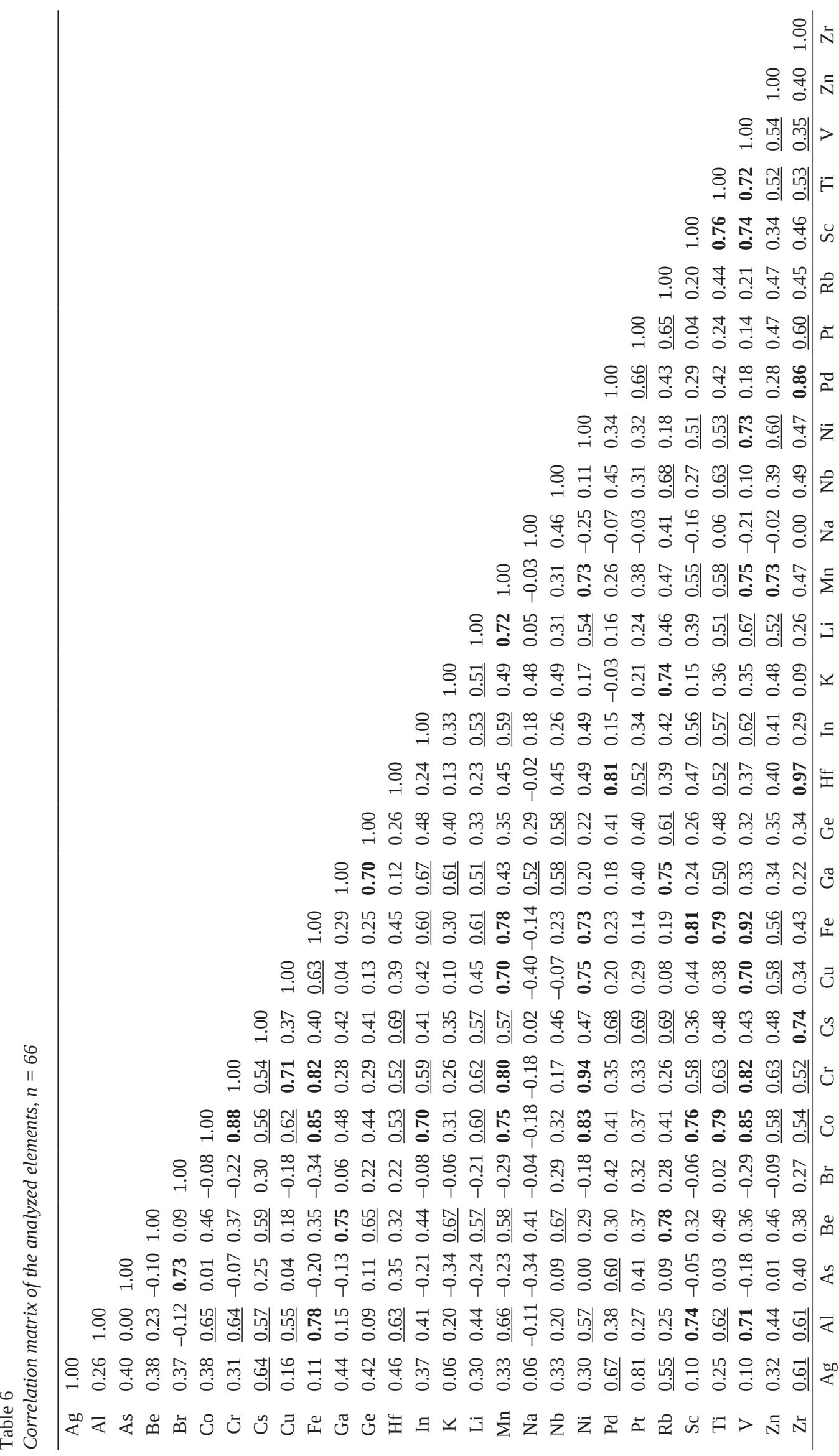


stability of the new synthetic variables, i.e., associations of elements. The dendrogram of the distances among the individual elements is presented in Fig. 5. Similar results were achieved by the application of multivariate factor analysis (Table 7). All of the obtained clusters correspond to the three obtained factors.

Factor 1 (Fig. 6) is a geogenic association composed of 13 elements: Fe, V, Cr, Co, Ni, Mn, Cu, $\mathrm{Al}, \mathrm{Sc}, \mathrm{Ti}, \mathrm{Li}, \mathrm{Zu}$ and In. The highest content of these elements is characteristic for the soil from the eastern (Vardar tectonic zone), southern and eastern parts (Pelagonian massif). In the eastern part of the area, Proterozoic gneisses and carbonates and Neogene felsic pyroclastites are the dominant geological formations. In the western part, the dominant geological formations are Proterozoic gneisses together with Proterozoic felsic plutonites and shales. Thus, the content of Fe reaches the highest content of $4.8 \%$ (median content is $1.6 \%$ ) in the eastern part of the region. The maximum contents of the other elements from F1 were also found in the mentioned areas of the region: $110 \mathrm{mg} / \mathrm{kg}$ for $\mathrm{V}$ (median value $33 \mathrm{mg} / \mathrm{kg}$ ), $540 \mathrm{mg} / \mathrm{kg}$ for Cr (median $15 \mathrm{mg} / \mathrm{kg}$ ), $36 \mathrm{mg} / \mathrm{kg}$ for Co (median of $5.0 \mathrm{mg} / \mathrm{kg}$ ), $450 \mathrm{mg} / \mathrm{kg}$ for $\mathrm{Ni}$ (median of $8.2 \mathrm{mg} / \mathrm{kg}$ ), $930 \mathrm{mg} / \mathrm{kg}$ for $\mathrm{Mn}$ (median of $300 \mathrm{mg} / \mathrm{kg}$ ), $38 \mathrm{mg} / \mathrm{kg}$ for $\mathrm{Cu}$ (median of $5.8 \mathrm{mg} / \mathrm{kg}$ ), $5.6 \%$ for Al (median of $2.2 \%$ ), $7.5 \mathrm{mg} / \mathrm{kg}$ for Sc (median of $2.4 \mathrm{mg} / \mathrm{kg}$ ), $0.27 \%$ for $\mathrm{Ti}$ (median of $0.15 \%$ ), $42 \mathrm{mg} / \mathrm{kg}$ for $\mathrm{Li}$ (median of $14 \mathrm{mg} / \mathrm{kg}$ ), $150 \mathrm{mg} / \mathrm{kg}$ for Zn (median of $45 \mathrm{mg} / \mathrm{kg}$ ) and $0.075 \mathrm{mg} / \mathrm{kg}$ for Co (median of $0.026 \mathrm{mg} / \mathrm{kg}$ ). Lower contents of the elements of this factor were found in soil from the central part of the region (villages of Budimirci, Staravina and Bešište, where Neogene felsic pyroclastites and volcanites are the dominant lithological formations).

Factor 2 (Fig. 7) is a geogenic association that is composed of eight elements: Pd, As, Zr, Pt, Ag, Hf, $\mathrm{Br}$ and $\mathrm{Cs}$. The presence of these elements is char-



Fig. 5. Dendrogram from the cluster analysis $(n=28)$. 
Table 7

Matrix of dominant rotated factor loadings $(n=66)$

\begin{tabular}{|c|c|c|c|c|}
\hline & F1 & F2 & F3 & Assoc. \\
\hline $\mathrm{Fe}$ & 0.94 & -0.03 & 0.12 & 90.1 \\
\hline V & 0.93 & -0.06 & 0.13 & 89.1 \\
\hline $\mathrm{Cr}$ & 0.90 & 0.15 & 0.10 & 85.1 \\
\hline Co & 0.87 & 0.22 & 0.23 & 86.7 \\
\hline $\mathrm{Ni}$ & 0.85 & 0.17 & 0.00 & 76.1 \\
\hline Mn & 0.83 & 0.07 & 0.35 & 81.5 \\
\hline $\mathrm{Cu}$ & 0.79 & 0.11 & -0.14 & 65.7 \\
\hline $\mathrm{Al}$ & 0.77 & 0.24 & 0.01 & 65.9 \\
\hline Sc & 0.76 & 0.10 & 0.08 & 59.0 \\
\hline $\mathrm{Ti}$ & 0.68 & 0.22 & 0.38 & 66.0 \\
\hline $\mathrm{Li}$ & 0.63 & -0.03 & 0.46 & 61.6 \\
\hline $\mathrm{Zn}$ & 0.61 & 0.19 & 0.34 & 52.1 \\
\hline In & 0.59 & 0.02 & 0.46 & 56.8 \\
\hline Pd & 0.23 & 0.89 & 0.09 & 85.0 \\
\hline As & -0.18 & 0.80 & -0.27 & 74.4 \\
\hline $\mathrm{Zr}$ & 0.44 & 0.78 & 0.13 & 81.8 \\
\hline $\mathrm{Pt}$ & 0.19 & 0.72 & 0.30 & 64.8 \\
\hline $\mathrm{Ag}$ & 0.14 & 0.72 & 0.31 & 63.7 \\
\hline $\mathrm{Hf}$ & 0.47 & 0.71 & 0.06 & 73.4 \\
\hline $\mathrm{Br}$ & -0.35 & 0.69 & 0.06 & 60.0 \\
\hline Cs & 0.44 & 0.64 & 0.38 & 75.2 \\
\hline Ga & 0.20 & 0.09 & 0.87 & 80.7 \\
\hline $\mathrm{Rb}$ & 0.14 & 0.41 & 0.82 & 86.5 \\
\hline $\mathrm{Be}$ & 0.28 & 0.19 & 0.82 & 78.2 \\
\hline K & 0.22 & -0.14 & 0.79 & 69.6 \\
\hline $\mathrm{Na}$ & -0.29 & -0.15 & 0.72 & 62.3 \\
\hline $\mathrm{Nb}$ & 0.09 & 0.37 & 0.71 & 64.5 \\
\hline $\mathrm{Ge}$ & 0.18 & 0.30 & 0.66 & 56.4 \\
\hline Prp. Totl & 33.4 & 18.7 & 19.8 & 71.9 \\
\hline Eigen Val & 12.10 & 4.43 & 3.59 & \\
\hline Expl. Var & 9.34 & 5.24 & 5.54 & \\
\hline
\end{tabular}

F - load factor values; F1, F2 and F3 - load factor values for each appropriate factor 1, 2 and 3; Assoc. - association (\%); Prp. Totl - total variance of the system; Eigen Val - Eingene values; Expl. Var - variance of the special component.

acteristic of the central part of Mariovo, around the village of Bešište, and the eastern part around Nidže. This factor is geogenic because the distribution is related to geology, i.e., the highest contents of these elements are observed in the areas of Proterozoic plutonites and gneisses, Neogene felsic pyroclastites, sediments and volcanites, Proterozoic gneisses and carbonates. Thus, the content of Pd reaches its highest content of $1.6 \mathrm{mg} / \mathrm{kg}$ (median content is $0.13 \mathrm{mg} / \mathrm{kg}$ ) in the eastern part of the region. The maximum contents of the other elements from F2 were also found in the mentioned areas of the region: $100 \mathrm{mg} / \mathrm{kg}$ for As (median value $13 \mathrm{mg} / \mathrm{kg}$ ), $98 \mathrm{mg} / \mathrm{kg}$ for Zr (median $11 \mathrm{mg} / \mathrm{kg}$ ), $0.61 \mathrm{mg} / \mathrm{kg}$ for Pt (median of $0.19 \mathrm{mg} / \mathrm{kg}$ ), $1.9 \mathrm{mg} / \mathrm{kg}$ for $\mathrm{Ag}$

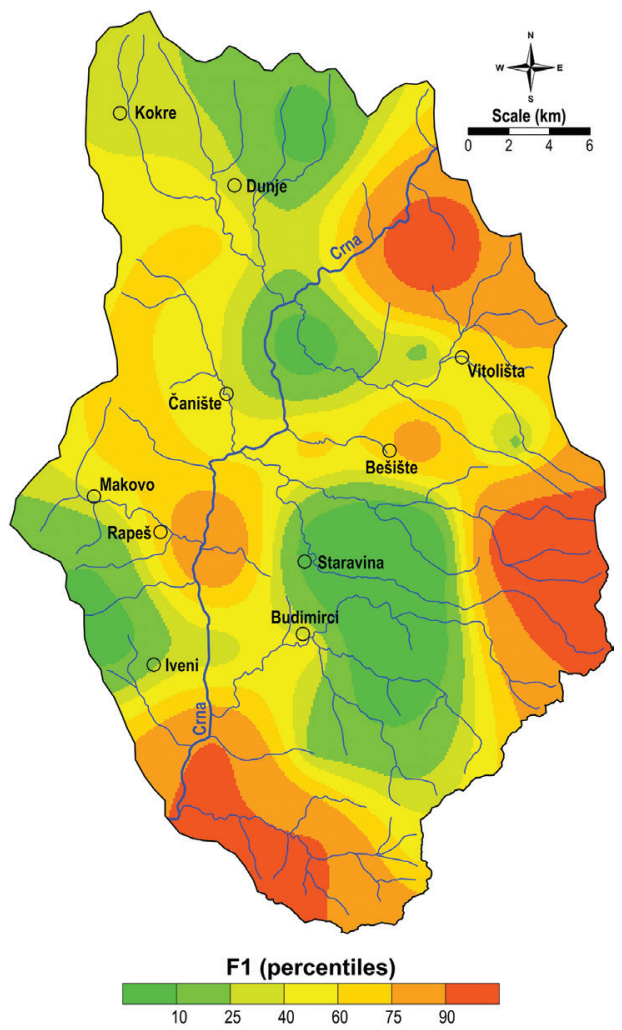

Fig. 6. Spatial distribution of factor scores of Factor 1 (Fe-VCr-Co-Ni-Mn-Cu-Al-Sc-Ti-Li-Zn-In).

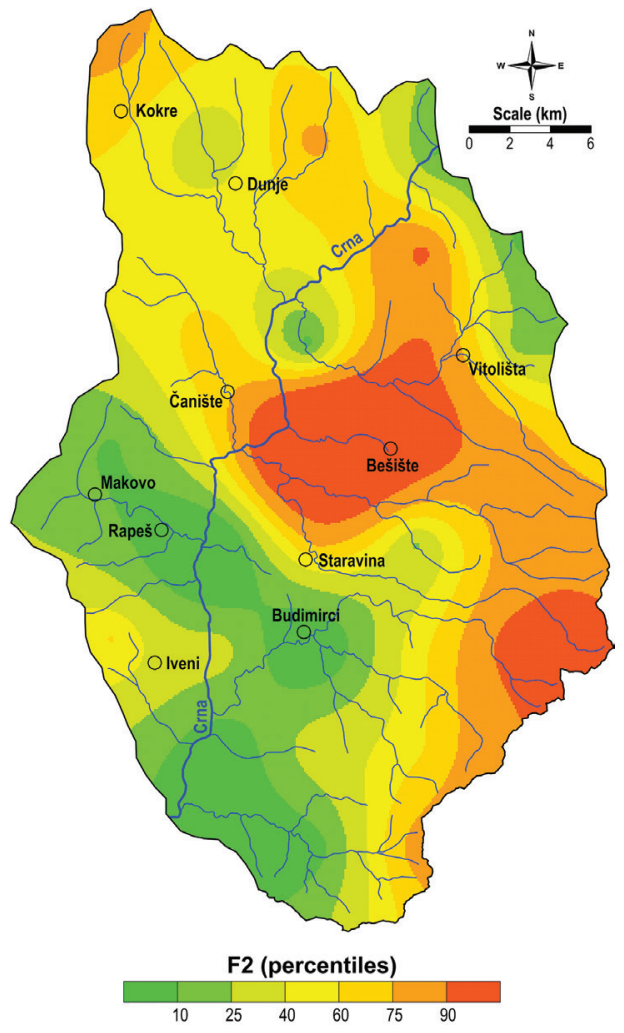

Fig. 7. Spatial distribution of factor scores of Factor 2 (Pd-AsZr-Pt-Ag-Hf-Br-Cs). 


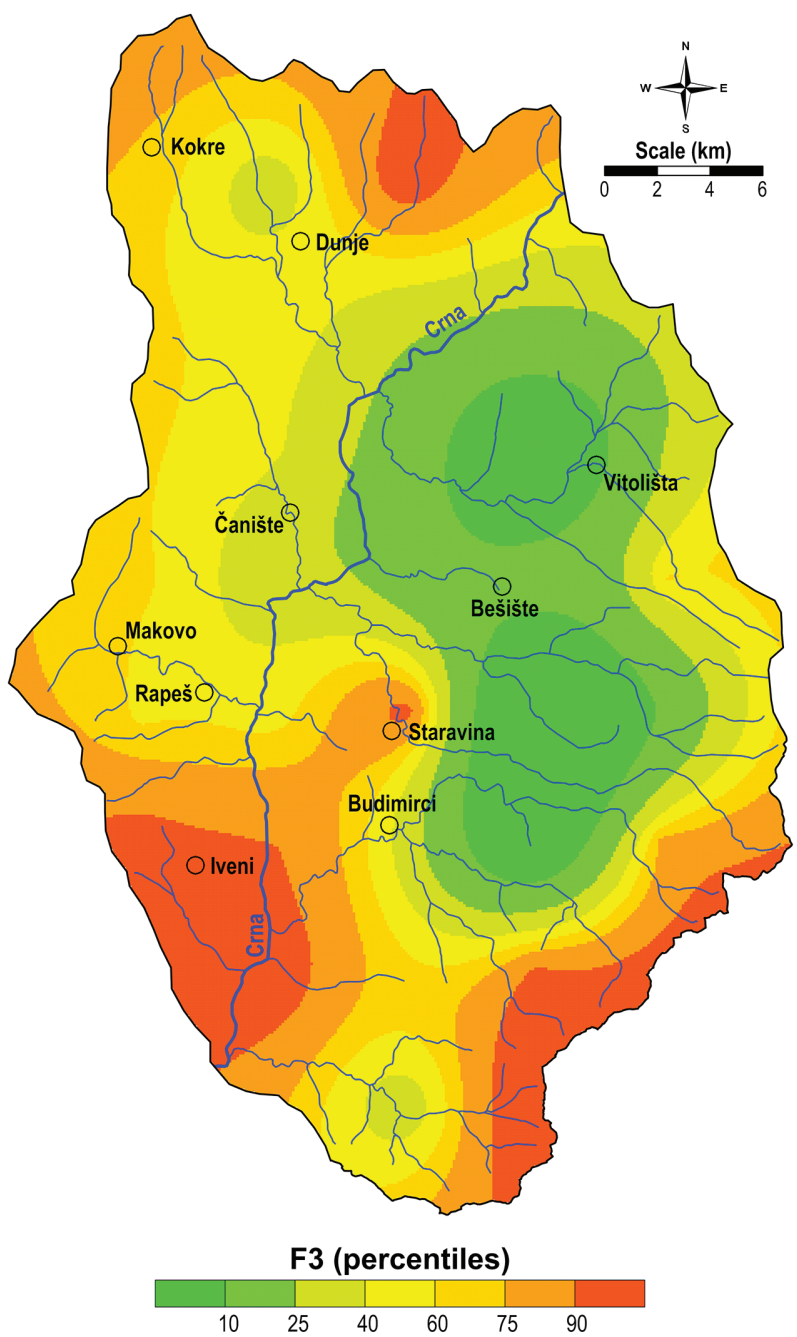

Fig 8. Spatial distribution of factor scores of Factor 3 (Ga-RbBe-K-Na-Nb-Ge).

(median of $0.51 \mathrm{mg} / \mathrm{kg}$ ), $2.3 \mathrm{mg} / \mathrm{kg}$ for $\mathrm{Hf}$ (median of $0.38 \mathrm{mg} / \mathrm{kg}$ ), $18 \mathrm{mg} / \mathrm{kg}$ for $\mathrm{Br}$ (median of $1.5 \mathrm{mg} / \mathrm{kg}$ ) and $26 \mathrm{mg} / \mathrm{kg}$ for Cs (median of $2.7 \mathrm{mg} / \mathrm{kg}$ ). Lower distributions of the elements from F2 are in the south-western part of Mariovo, in the samples from the area where Proterozoic gneisses and felsic plutonites are the dominant geological formations.

Factor 3 represents the geogenic association that includes Ga, Rb, Be, K, Na, Nb and Ge. The spatial distribution maps of the factor scores of Factor 3 are given in Fig. 8. The presence of elements in higher contents is mostly in the soil samples from the southern part of the region and from the small area of the northern part with Proterozoic and Mesozoic carbonates and Proterozoic shales, gneisses and felsic plutonites. The maximum median content of $\mathrm{Ga}$ from these areas is $31 \mathrm{mg} / \mathrm{kg}$



Fig. 9. Spatial distribution of rare earth elements (Ce, Dy, Er, Eu, Gd, Ho, La, Nd, Pr, Sm, Tb, Tm and Yb).

(median of $11 \mathrm{mg} / \mathrm{kg}$ ), $\mathrm{Rb}$ is $160 \mathrm{mg} / \mathrm{kg}$ (median $40 \mathrm{mg} / \mathrm{kg}$ ), Be is $6.7 \mathrm{mg} / \mathrm{kg}$ (median $2.0 \mathrm{mg} / \mathrm{kg}$ ), $\mathrm{K}$ is $2.4 \%$ (median $1.3 \%$ ), $\mathrm{Na}$ is $1.9 \%$ (median $0.95 \%$ ), $\mathrm{Nb}$ is $25 \mathrm{mg} / \mathrm{kg}$ (median $40 \mathrm{mg} / \mathrm{kg}$ ) and Ge is $1.7 \mathrm{mg} / \mathrm{kg}$ (median $0.46 \mathrm{mg} / \mathrm{kg}$ ).

Fourteen rare-earth elements (Ce, Dy, Er, Eu, Gd, Ho, La, Nd, Pr, Sm, Tb, Tm, and Yb) were also determined by ICP-MS (Fig. 9). It can be noted that the highest values exist in the areas dominated by Neogene clastic sediments, Proterozoic shales and felsic plutonites in the western part of the Mariovo region, which is a part of the Pelagonian massif known for increased contents of REEs (Stafilov and Šajn, 2016, 2019). Potential deposits of REEs in mineral ore deposits can be divided into primary and secondary. Primary deposits are those formed by magmatic, hydrothermal and/or metamorphic 



Fig. 10. Spatial distribution maps of the potentially toxic elements not included in the factors (Bi, $\mathrm{Cd}, \mathrm{Pb}, \mathrm{Sb}, \mathrm{Sn}$ and $\mathrm{Tl})$.

processes. REEs in soils are predominately concentrated in minerals, such as fluorocarbonates, phosphates, silicates and oxides (Al-Ani et al., 2018).

As previously mentioned, from a total of 68 analyzed elements, the factor analysis reduced them to 28 elements; 14 elements are associated with the group of rare earth elements (REEs), while the remaining 16 elements (Ba, Bi, Ca, Cd, I, Mg, Mo, P, $\mathrm{Pb}, \mathrm{Sb}, \mathrm{Sn}, \mathrm{Sr}, \mathrm{Ta}, \mathrm{Tl}, \mathrm{W}$ and $\mathrm{Y}$ ) were eliminated due to their lack of participation in the association. Spatial distribution maps of the potentially toxic elements not included in the factors $(\mathrm{Bi}, \mathrm{Cd}, \mathrm{Pb}, \mathrm{Sb}$, 
Sn and $\mathrm{Tl}$ ) are given in Fig. 10. Especially noteworthy is the increased content of As, Sb and $\mathrm{Tl}$ in the southwestern part of the studied area, the western part of Mount Nidže and the western parts of Mount Kožuf, where the Allchar mine operates, known for its large reserves of arsenic, antimony and thallium mineralization. This reflects the increased content of As up to $100 \mathrm{mg} / \mathrm{kg}$ (increasing the action value to almost 2×), of Sb $1.6 \mathrm{mg} / \mathrm{kg}$ and of $\mathrm{Tl}$ up to $1.2 \mathrm{mg} / \mathrm{kg}$ in the surrounding soils (Bačeva et al., 2014; Stafilov and Šajn, 2016, 2019; Jovanovski et al., 2018, 2019).

\section{CONCLUSION}

Examination of the distribution of 58 chemical elements in soil samples from the Mariovo region, North Macedonia, was performed. Soil samples collected from 60 locations were analyzed by using two spectroscopic techniques: inductively coupled plasma atomic emission spectrometry (ICPAES) for major elements and inductively coupled plasma mass spectrometry (ICP-MS) for trace elements. Factor analysis of the major elements gives three geogenic factors and their spatial distributions showed that their occurrences are geologically determined. In factor analysis, the distribution is reduced to three synthetic variables (F1-F3): F1 (Fe, V, Cr, Co, Ni, Mn, Cu, Al, Sc, Ti, Li, Zn and In); F2 (Pd, As, Zr, Pt, Ag, Hf, Br and Cs); and F3 (Ga, Rb,
$\mathrm{Be}, \mathrm{K}, \mathrm{Na}, \mathrm{Nb}$ and $\mathrm{Ge}$ ). Factor 1 is a geogenic association composed of 13 elements: $\mathrm{Fe}, \mathrm{V}, \mathrm{Cr}, \mathrm{Co}, \mathrm{Ni}$, $\mathrm{Mn}, \mathrm{Cu}, \mathrm{Al}, \mathrm{Sc}, \mathrm{Ti}, \mathrm{Li}, \mathrm{Zu}$ and In. Their highest content was found in soil from the eastern part of the region, which belongs to the Vardar tectonic zone, and southern and eastern parts, which belong to the Pelagonian massif where Proterozoic gneisses and carbonates and Neogene felsic pyroclastites are the dominant geological formations. In the eastern part, the dominant geological formations are Proterozoic gneisses together with Proterozoic felsic plutonites and shales. Factor 2 is a geogenic association composed of eight elements (Pd, As, Zr, Pt, Ag, Hf, Br and Cs). Their presence is characteristic of the central part of Mariovo and their highest contents were observed in the areas of Proterozoic plutonites and gneisses, Neogene felsic pyroclastites, sediments and volcanic rocks, and Proterozoic gneisses and carbonates. Factor 3 represents the geogenic association that includes $\mathrm{Ga}, \mathrm{Rb}, \mathrm{Be}, \mathrm{K}, \mathrm{Na}, \mathrm{Nb}$ and $\mathrm{Ge}$. The presence of elements in higher contents is mostly in the soil samples from the southern part of the region and the small area of the northern part with Proterozoic and Mesozoic carbonates, and Proterozoic shales, gneisses and felsic plutonites.

\section{Acknowledgements}

The authors wish to thank two anonymous reviewers for their constructive reviews and suggestions, which improved the quality of the manuscript.

\section{REFERENCES}

Acton, Q.A. (Ed.). 2013. Issue in Environmental Health and Pollution. Scholarly Editions, Atlanta, Georgia, 1174 pp.

Al-Ani, T., Molnár, F., Lintinen, P., Leinonen, S. 2018. Geology and mineralogy of rare earth elements deposits and occurrences in Finland. Minerals 8 (8), 356, https://doi. org/10.3390/min8080356.

Bačeva, K., Stafilov, T., Šajn R., Tănăselia, C. 2012. Moss biomonitoring of air pollution with heavy metals in the vicinity of a ferronickel smelter plant. Journal of Environmental Science and Health A47 (4), 645-656, https://doi.org/10.10 80/10934529.2012.650587.

Bačeva, K., Stafilov, T., Šajn, R., Tănăselia, C., Makreski, P. 2014. Distribution of chemical elements in soils and stream sediments in the area of abandoned Sb-As-Tl Allchar mine, Republic of Macedonia. Environmental Research 133, 7789, https://doi.org/10.1016/j.envres.2014.03.045.

Balabanova, B., Stafilov, T., Bačeva, K., Šajn, R. 2010. Biomonitoring of atmospheric pollution with heavy metals in the copper mine vicinity located near Radoviš, Republic of Macedonia. Journal of Environmental Science and Health A45 (12), 1504-15181, https://doi.org/10.1080/10934529.2010.506097.
Balabanova, B., Stafilov, T., Šajn, R., Bačeva, K. 2013. Spatial distribution and characterization of some toxic metals and lithogenic elements in topsoil and subsoil from copper mine environs. International Journal of Environmental Protection 3 (9), 1-9.

Davidson, C.M., Urquhart, G.J., Ajmone-Marsan, F., Biasioli, M., Duarte, A.D., Díaz-Barrientos, E., Grčman, H., Hossack, L., Hursthouse, A.S., Madrid, L., Rodrigues, S., Zupan, M. 2006. Fractionation of potentially toxic elements in urban soils from five European cities by means of a harmonised sequential extraction procedure. Analytica Chimica Acta 565, 63-72, https://doi.org/10.1016/j. aca.2006.02.014.

Diawara, M.M., Litt, J.S., Unis, D., Alfonso, N., Martinez, L.A., Crock, J.G., Smith, D.B., Carsella, J. 2006. Arsenic, cadmium, lead, and mercury in surface soils, Pueblo, Colorado: implications for population health risk. Environmental Geochemistry and Health 28, 297-315, https://doi. org/10.1007/s10653-005-9000-6.

Dumurdzanov, N., Serafimovski, T., Burchfiel, B.C. 2004. Evolution of the Neogene-Pleistocene Basins of Macedo- 
nia. Geological Society of America, Digital Map and Chart Series 1, 20 pp.

Dumurdžanov, N., Krstić, N., Mihajlović, Dj., Ognjanova-Rumenova, N., Petrov., G. 2003. New data on stratigraphy of the Neocene and Pleistocene in Mariovo, Macedonia. Geologica Macedonica 17, 43-52.

Dumurdzanov, N., Petrov, G., Stojanova, V. 2013. The geology and geotectonic setting of the Kozuf Mountain area (R. Macedonia). Proceedings of the $1^{\text {st }}$ International Workshop on the Project "Environmental impact assessment of the Kozuf metallogenic district in southern Macedonia in relation to groundwater resources, surface waters, soils and socio-economic consequences (ENIGMA)", 10 October 2013, Kavadarci, 23-32.

Facchinelli, A., Sacchi, E., Mallen, L. 2001. Multivariate statistical and GIS-based approach to identify heavy metal sources in soils. Environmental Pollution 114 (3), 313-324, https://doi.org/10.1016/S0269-7491(00)00243-8.

Filipovski, G., Andreevski, M., Vasilevski, K., Milevski, I., Markoski, M., Mitkova, T., Mitrikeski, J., Mukaetov, D., Petkovski, D. 2015. Pedological (Soil) Map. Sts Cyril and Methodius University, Skopje.

ISO 14869-1:2001. 2001. Soil quality - Dissolution for the determination of total element content - Part 1: Dissolution with hydrofluoric and perchloric acids. International Organization for Standardization, Geneva, 5 pp.

Lazarevski, A. 1993. Climate in Macedonia. Kultura, Skopje, 253 pp.

Kabata-Pendias, A., Mukherjee, A.B. 2007. Trace Elements from Soil to Human. Springer, Heidelberg, 255 pp., https:// doi.org/10.1007/978-3-540-32714-1.

Koteski, C. 2013. Thematic Atlas of the Mariovo and Raechka Valleys in Macedonia. Lambert Academic Publishing, Saarbrucken, 222 pp.

Koteski, C., Jakovlev, Z., Mitreva, E., Angelkova, T., Kitanov, V. 2012. Aspects of land and their distribution in Mariovo and Raječka Valley in Republic of Macedonia. XII Conference on biotechnology with international participation, 6-7 April 2012, Cacak, Serbia, 1-6.

Peplow, D. 1999. Environmental Impacts of Mining in Eastern Washington. Center for Water and Watershed Studies Fact Sheet, University of Washington, Seattle, 2 pp.

Petrušev, E., Stolić, N., Šajn, R., Stafilov, T. 2021. Geological characteristics of the Republic of North Macedonia. Geologica Macedonica 35 (1), 49-58, https://doi.org/10.46763/ GEOL21351372049ep.

Reimann, C., Filzmoser, P., Garrett, R.G. 2002. Factor analysis applied to regional geochemical data: problems and possibilities. Applied Geochemistry 17 (3), 185-206, https://doi. org/10.1016/S0883-2927(01)00066-X.

Reimann, C., Äyräs, M., Chekushin, V.A., Bogatyrev, I.V., Boyd, R., Caritat, P., Dutter, R., Finne, T.E., Halleraker, J.H., Jæger, Ø., Kashulina, G., Lehto, O., Niskavaar, H., Pavlov, V.A., Räisänen, M.L., Strand, T., Volden, T. 2011. Environmental Geochemical Atlas of the Central Barents Region. Geological Survey of Finland and Geological Survey of Norway, Trondheim, $743 \mathrm{pp}$.
Salminen, R., Batista, M.J., Bidovec, M., Demetriades, A., De Vivo, B., De Vos, W., Duris, M., Gilucis, A., Gregorauskiene, V., Halamic, J., Heitzmann, P., Jordan, G., Klaver, G., Klein, P., Lis, J., Locutura, J., Marsina, K., Mazreku, A., O’Connor, P.J., Olsson, S.Å., Ottesen, R.T., Petersell, V., Plant, J.A., Reeder, S., Salpeteur, I., Sandström, H., Siewers, U., Steenfelt, A., Tarvainen, T. 2005. Geochemical atlas of Europe, Part 1, Background Information, Methodology and Maps. Geological Survey of Finland, Espoo, 526 pp.

Salomons, W., Förstner, U., Mader, P. (Eds). 2012. Heavy Metals: Problems and Solutions. Springer Science \& Business Media, Heidelberg, 412 pp.

Serafimovska, J.M., Arpadjan, S., Stafilov, T., Tsekova, K. 2013. Study of the antimony species distribution in industrially contaminated soils. Journal of Soils and Sediments 13 (2), 294-303, https://doi.org/10.1007/s11368-012-0623-9.

Stafilov, T., Šajn, R. 2016. Geochemical Atlas of the Republic of Macedonia. Faculty of Natural Sciences and Mathematics, Skopje, 239 pp.

Stafilov, T., Šajn, R. 2019. Spatial distribution and pollution assessment of heavy metals in soil from the Republic of North Macedonia. Journal of Environmental Science and Health A54 (14), 1457-1474, https://doi.org/10.1080/10934529. 2019.1656498.

Stafilov, T., Šajn, R., Ahmeti, L. 2019. Geochemical characteristics of soil of the city of Skopje, Republic of Macedonia. Journal of Environmental Science and Health A54 (10), 972-987, https://doi.org/10.1080/10934529.2019. 1620042.

Stafilov, T., Šajn, R., Alijagić, J. 2013. Distribution of arsenic, antimony and thallium in soil in Kavadarci and its surroundings, Republic of Macedonia. Soil and Sediment Contamination: An International Journal 22, 105-118, https:// doi.org/10.1080/15320383.2012.714425.

Stafilov, T., Šajn, R., Arapčeska, M., Kungulovski, I., Alijagić, J. 2018. Geochemical properties of topsoil around the coal mine and thermoelectric power plant, Journal of Environmental Science and Health A53 (9), 793-808, https://doi. org/10.1080/10934529.2018.1445076.

Stafilov, T., Šajn, R., Boev, B., Cvetković, J., Mukaetov, D., Andreevski, M., Lepitkova, S. 2010b. Distribution of some elements in surface soil over the Kavadarci Region, Republic of Macedonia. Environmental Earth Sciences 61, 15151530, https://doi.org/10.1007/s12665-010-0467-9.

Stafilov, T., Šajn, R., Pančevski, Z., Boev, B., Frontasyeva, M.V., Strelkova, L.P. 2010a. Heavy metal contamination of topsoils around a lead and zinc smelter in the Republic of Macedonia. Journal of Hazardous Materials 175 (1-3), 896-914, https://doi.org/10.1016/j.jhazmat.2009.10.094.

Temovski, M. 2013. Characteristics of hypogenic karstification and occurrence of hypogenic karst in Republic of Macedonia. Geografski razgledi 47, 11-29.

Wang, Y., Yang, L., Kong, L., Liu, E., Wang, L., Zhu, J. 2015. Spatial distribution, ecological risk assessment and source identification for heavy metals in surface sediments from Dongping Lake, Shandong, East China. Catena 125, 200205, https://doi.org/10.1016/j.catena.2014.10.023. 